\title{
BOUNDED-ANALYTIC SEQUENT CALCULI AND EMBEDDINGS FOR HYPERSEQUENT LOGICS
}

\author{
AGATA CIABATTONI, TIMO LANG, AND REVANTHA RAMANAYAKE
}

\begin{abstract}
A sequent calculus with the subformula property has long been recognised as a highly favourable starting point for the proof theoretic investigation of a logic. However, most logics of interest cannot be presented using a sequent calculus with the subformula property. In response, many formalisms more intricate than the sequent calculus have been formulated. In this work we identify an alternative: retain the sequent calculus but generalise the subformula property to permit specific axiom substitutions and their subformulas. Our investigation leads to a classification of generalised subformula properties and is applied to infinitely many substructural, intermediate, and modal logics (specifically: those with a cutfree hypersequent calculus). We also develop a complementary perspective on the generalised subformula properties in terms of logical embeddings. This yields new complexity upper bounds for contractive-mingle substructural logics and situates isolated results on the so-called simple substitution property within a general theory.
\end{abstract}

\$1. Introduction. What concepts are essential to the proof of a given statement? This is a fundamental and long-debated question in logic since the time of Leibniz, Kant, and Frege, when a broad notion of analytic proof was formulated to mean truth by conceptual containments, or purity of method in mathematical arguments.

The familiar Hilbert-style proof systems are excellent for many purposes including defining a logic and a notion of proof. However they offer few insight concerning analyticity because of their reliance on the inference rule of modus ponens. The issue is that the conclusion $B$ of modus ponens may be quite unrelated to the $A$ that occurs in its premises $A \rightarrow B$ and $A$. In 1935, Gentzen [20] showed how to address this weakness of the Hilbert systems by placing the logical language within a meta-logical (structural) language. Specifically, he introduced a new type of proof system called the sequent calculus built from sequents $\Gamma \Rightarrow \Delta$ where $\Gamma$ and $\Delta$ are lists/multisets of logical formulas. Enriching the logical language with a structural language enabled him to state and prove the famous cut-elimination theorem - this is a constructive procedure that eliminates all applications of the cut rule from a given proof. The cut rule is a generalisation of modus ponens and it is the only non-analytic rule in the classical and intuitionistic sequent calculi. So from cut-elimination it follows that every provable formula has a proof respecting the subformula property (i.e., every formula in the proof is a subformula of the theorem). Gentzen later used this property to give a proof of the consistency of arithmetic. This cemented

Received May 11, 2020.

2020 Mathematics Subject Classification. Primary 03F03, Secondary 03F07, 03F52.

Key words and phrases. sequent calculus, subformula property, cut-elimination, hypersequent calculus, substructural logics, modal logics, non-classical logics, structural proof theory.

(C) The Author(s), 2021. Published by Cambridge University Press. This is an Open Access article, distributed under the terms of the Creative Commons Attribution licence (https://creativecommons.org/licenses/by/4.0/), which permits unrestricted re-use, distribution, and reproduction in any medium, provided the original work is properly cited. 
the significance of the sequent calculus, and analyticity came to be seen as nearsynonymous with the subformula property.

The decades following Gentzen's work saw an explosion of results establishing the subformula property via cut-elimination for sequent calculi for various logics. The subformula property is a significant restriction on the proof search space which can be exploited to establish metalogical results (e.g., consistency, decidability, complexity, interpolation, and disjunction properties) and for automated reasoning. Nevertheless, already from the 1960s it was observed (for example, Mints [36]) that the formalism of the sequent calculus was not expressive enough to provide the subformula property for most logics of interest. The response of the structural proof theory community was to obtain the subformula property by developing new exotic proof formalisms (e.g., hypersequent, bunched, nested sequent, display, labelled calculi, tree-hypersequent, and many more) that further extend the structural language of the sequent calculus.

Introduced independently by Mints [36], Pottinger [38], and Avron [2], the hypersequent calculus is one of the most successful such formalisms. A hypersequent goes just one step further in the sense that it is a multiset of Gentzen's sequents, denoted as $\Gamma_{1} \Rightarrow \Delta_{1}|\cdots| \Gamma_{n} \Rightarrow \Delta_{n}$. Hypersequent calculi with the subformula property have been presented for many non-classical logics that could not be provided this property in the sequent calculus. Especially noteworthy are the uniform and modular extensions of base systems for commutative substructural logics [12] and modal logics [28, 29, 31].

However, the price to be paid for moving to an exotic proof formalism - even in the simple case of hypersequents - is having to tame its richer structural language in order to use the proof calculus to prove metalogical results.

Rather than privileging the subformula property and developing exotic formalisms that provide it, we propose an alternative. We stay with the sequent calculus - thus benefitting from the simplicity of its structural language - and identify generalisations of the subformula property that can be useful to prove metalogical results. In a nutshell, we tackle the question:

What are some useful generalisations of the subformula property for the sequent calculus?

Isolated proposals for perturbing the subformula property have been presented for specific families of logics. In contrast, we propose a hierarchy of generalisations of the subformula property that are logic- and language-independent. Our interests are methodological and also aim for concrete results. Specifically, we obtain generalised subformula properties in the sequent calculus for the commutative substructural logics in [12] and for the modal logics in [29], starting from analytic hypersequent calculi for these logics.

Our work can be seen from two perspectives.

(I) Proof theory: We generalise the subformula property by permitting subformulas of specific (i.e., restricted) substitutions of the logic's axioms. From this perspective, proofs satisfying the subformula property are the lower limit of our classification, while proofs with arbitrary cuts are the upper limit. We achieve this result by transforming hypersequent calculi into sequent calculi such that the cut-formulas in the latter are restricted to axioms whose 
propositional variables are substituted using variables, or formulas, or lists of formulas (without, or possibly with, repetitions). In each case, the formulas that are used must occur in the end formula. The ensuing sequent calculi are called, respectively, variable-analytic, formula-analytic, set-analytic, and multiset-analytic. Collectively they are called bounded-analytic sequent calculi. As a corollary we obtain a new syntactic proof of a well-known result [17]: the subformula property of the sequent calculus for the modal logic S5.

(II) Embeddings: We characterise axiomatic extensions of a base logic in terms of a function embedding it into the base logic. The form of the functions determines the degree of boundedness. This perspective is helpful for metalogical argumentation since we are no longer constrained by the minute syntactic details. As a corollary we obtain new decidability and complexity upper bounds for a large class of substructural logics with contraction and mingle (such as, e.g., UML [34]) and hence also decidability of the equational theory of the corresponding classes of residuated lattices [18]. Moreover we also obtain sharper embeddings from intermediate logics into intuitionistic logic, and situate within our theory scattered results from the 1980s on the simple substitution property [23].

1.1. Related work. Subformula property without cut-elimination. Beginning with Smullyan [43], several works have investigated cuts on subformulas of the end formula ('analytic cuts'). The resulting proofs are not cut-free but they do satisfy the subformula property. Takano's intricate proof [45] of the subformula property for S5 via analytic cuts belongs to this literature. D'Agostino and Mondadori [15] show that analytic cuts for classical logic can be used to gain a deterministic speedup in proof search. Fitting [17] proved that the sequent calculi of several modal logics possess the subformula property by a logic-specific semantic argument. Algebraic arguments were employed by Kowalski and Ono [27] to show that a sequent calculus for bi-intuitionistic logic has the analytic cut property. Avron and Lahav [6] give sufficient conditions for the subformula property in sequent calculi whose rules obey a certain shape.

Bezhanishvili and Ghilardi [10] investigate what can be said about a Hilbert system when the logic possesses a sequent calculus with the subformula property. In that work several modal logics were shown to possess the bounded proof property - this is a restriction on the modal complexity of formulas that need to appear in their Hilbert proofs (as a function of the formula being proved). The same algebraic approach is applied in [11] to show that analytic hypersequent calculi for intermediate logics satisfy a bounded property, this time with respect to the implicational complexity of formulas.

Generalisations of the subformula property have been investigated for specific families of logics. Avron [5] considers $s$ - $n$-analyticity in the context of paraconsistent logics, while Lellmann and Pattinson [32] introduce a generalisation in the context of conditional logics where a modal operator appended to propositional combinations of subformulas of the end formula is permitted, and obtain some tight complexity bounds. Lahav and Zohar [30] define a subformula property modulo the presence of leading negation symbols and provide a method for constructing sequent calculi with this property for sub-logics of a base logic. 
The above are certainly in the spirit of our work here. A point of difference with the above is that the generalisations proposed here are logic- and language-independent and provide a classification. We also provide a complementary perspective on this classification in terms of logical embeddings.

$A$ different solution to the absence of the subformula property is presented by Benzmüller [9] who obtains a cut-free proof calculus for quantified conditional logic by embedding their semantics in classical higher-order logic. This approach might make it possible to take advantage of existing theorem provers. However, it does not support a proof theoretic investigation of a logic because properties such as decidability, complexity, and proof structures are obscured or lost under the embedding into higher-order logic. Furthermore, although the higher-order calculus is cut-free, its proof rules contain higher-order variables that can be instantiated by arbitrary formulas ('cut-simulation').

Embeddings from axiomatic extensions to the base logic. Hosoi [23] introduced the notion of the simple substitution property. This was followed up by Sasaki [4042] who gives positive and negative criteria for this property to hold in various intermediate logics. The simple substitution property corresponds to variableaxiomatisations under our embedding perspective. Avellone et al. [1] present a semantic-based method and so-called selection functions to establish that certain intermediate logics are - in our terminology_variable-axiomatisations and (a variant of) formula-axiomatisations of intuitionistic logic. In contrast, our approach is not tailored to any specific logic or family of logics, and leads to a general theory encompassing proof theoretic and embedding perspectives. The simple substitution property and selection functions are specific instances in our classification.

The embedding functions that we present have a closed-form (explicit) definition, and their complexity depends solely on whether we are considering a set- or formula-axiomatisation. This makes it possible to use them in decidability and complexity arguments. Embeddings that satisfy fewer structural properties - for example, conservative translations in the sense of [16], which exist between most nonclassical logics (see Jerábek [24]) — are not so useful for deriving metalogical results.

Decidability and complexity. The decidability and 2EXPTIME complexity results for amenable extensions of $\mathbf{F L}_{e c m}$ presented here were first reported in the conference version of this paper, together with the EXPTIME complexity of $\mathbf{F L}_{\text {ecm }}$. This upper bound for $\mathbf{F L}_{e c m}$ improves on the 2EXPTIME given in St. John [44]. Note that a PSPACE lower bound for this logic appears in Horcík and Terui [21]. Ramanayake [39] showed that these decidability results hold even in the absence of mingle, and complexity upper bounds for these were given in Balasubramanian et al. [7].

1.2. Illustration of the key idea. Let us demonstrate how to transform a hypersequent calculus with the subformula property for propositional Gödel logici.e., intuitionistic logic extended with the axiom lin $:=(p \rightarrow q) \vee(q \rightarrow p)$-into a bounded-analytic sequent calculus. The hypersequent calculus we start with was obtained in [3] by adding the following rule to the hypersequent version HLJ of the sequent calculus $\mathbf{L} \mathbf{J}$ for intuitionistic logic:

$$
\frac{G\left|\Sigma_{1}, \Gamma_{1} \Rightarrow \Pi_{1} \quad G\right| \Sigma_{2}, \Gamma_{2} \Rightarrow \Pi_{2}}{G\left|\Sigma_{1}, \Gamma_{2} \Rightarrow \Pi_{1}\right| \Sigma_{2}, \Gamma_{1} \Rightarrow \Pi_{2}}(\mathrm{com}) .
$$


Consider the following derivation in $\mathbf{H L J}+(\mathrm{com})$ of $\Rightarrow F$. To highlight the key point, let us assume that the derivation contains a single instance of $(\mathrm{com})$.

$$
\begin{aligned}
& \pi_{1} \quad \pi_{2} \\
& \frac{\Sigma_{1}, \Gamma_{1} \Rightarrow \Pi_{1} \quad \Sigma_{2}, \Gamma_{2} \Rightarrow \Pi_{2}}{\Sigma_{1}, \Gamma_{2} \Rightarrow \Pi_{1} \mid \Sigma_{2}, \Gamma_{1} \Rightarrow \Pi_{2}}(\mathrm{com}) \\
& \frac{\Gamma^{\prime} \Rightarrow \Pi^{\prime} \mid \Gamma^{\prime} \Rightarrow \Pi^{\prime}}{\Gamma^{\prime} \Rightarrow \Pi^{\prime}}(\mathrm{ec}) \\
& \Rightarrow F \text {. }
\end{aligned}
$$

From this we can obtain the following sequent derivation in LJ. Note that for $\Gamma=\left\{A_{1}, \ldots, A_{n}\right\}$ we write $\wedge \Gamma$ to mean $A_{1} \wedge \cdots \wedge A_{n}$.

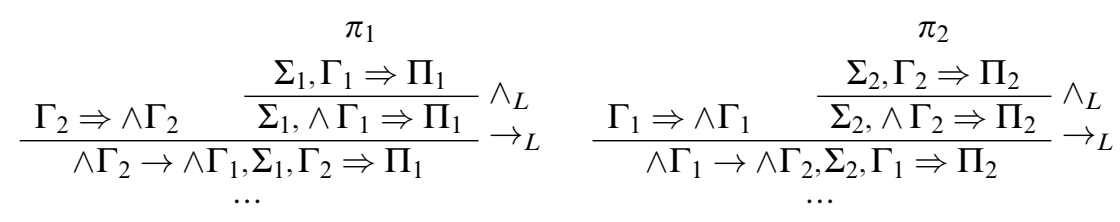

$$
\begin{aligned}
& \frac{\wedge \Gamma_{2} \rightarrow \wedge \Gamma_{1}, \Gamma^{\prime} \Rightarrow \Pi^{\prime} \quad \wedge \Gamma_{1} \rightarrow \wedge \Gamma_{2}, \Gamma^{\prime} \Rightarrow \Pi^{\prime}}{\left(\wedge \Gamma_{2} \rightarrow \wedge \Gamma_{1}\right) \vee\left(\wedge \Gamma_{1} \rightarrow \wedge \Gamma_{2}\right), \Gamma^{\prime} \Rightarrow \Pi^{\prime}} \vee_{L} \\
& \left(\wedge \Gamma_{2} \rightarrow \wedge \Gamma_{1}\right) \vee\left(\wedge \Gamma_{1} \rightarrow \wedge \Gamma_{2}\right) \Rightarrow F .
\end{aligned}
$$

By the subformula property in the hypersequent calculus, $\Gamma_{1}$ and $\Gamma_{2}$ are multisets consisting of subformulas of $F$.

We call $\sigma($ lin $)=\left(\wedge \Gamma_{2} \rightarrow \wedge \Gamma_{1}\right) \vee\left(\wedge \Gamma_{1} \rightarrow \wedge \Gamma_{2}\right)$ a multiset-substitution because each propositional variable in lin is substituted by a conjunction, possibly with repetition, of subformulas from the end formula $F$.

By iterating this argument, if a formula $F$ is a theorem of Gödel logic then there is some sequent $\sigma_{1}(\mathbf{l i n}), \ldots, \sigma_{n}(\mathbf{l i n}) \Rightarrow F$ derivable in $\mathbf{L J}$ such that each $\sigma_{i}$ is a multisetsubstitution. By applying the cut-rule it is easily seen that the reverse direction holds as well. As a consequence, we say that Gödel logic is multiset-axiomatisable over intuitionistic logic.

Exploiting the weakening and contraction rules in $\mathbf{L J}$ we can show that Gödel logic is in fact set-axiomatisable over intuitionistic logic (a set-substitution maps $p$ and $q$ to conjunctions, without repeats, of subformulas of $F$ ). In fact, we can improve this to formula-axiomatisable (a formula-substitution maps $p$ and $q$ to subformulas of $F$ ). Set- and formula-axiomatisations each give rise to an embedding into the base logic. This is the embedding perspective.

Alternatively, we have that $\mathbf{L J}$ extended by cuts on formulas of the shape $\sigma(\mathbf{l i n})$ with $\sigma$ a multiset/set/formula-substitution is a sequent calculus sound and complete for Gödel logic. Every formula in a derivation will then be a subformula of such $\sigma($ lin). This is the proof theoretic perspective.

1.3. Organisation of the paper. Preliminary notions are introduced in Section 2. Section 3 provides a formal introduction of the two perspectives. In Section 4 we 
discuss the key notion of disjunction form for a hypersequent rule and show how it leads to multiset-axiomatisations/analyticity. Next we show how to compute a disjunction form from each rule in an analytic hypersequent calculus (Section 5). As a consequence, substructural and intermediate logics are multiset-axiomatisable over, respectively, $\mathbf{F L}_{\mathbf{e}}$ and intuitionistic logic. Moreover, each such logic possesses a multiset-analytic sequent calculus. In Section 6 we show how the property can be strengthened in the presence of certain structural rules. We exploit these stronger forms of boundedness to establish complexity results, and show how interpolation is related to variable-analyticity. In Section 7 we show how the theory extends to modal logics.

This paper is an extension of work presented in [14]. The complementary perspective via embeddings, and the study of stronger forms of boundedness is new. Furthermore, the method is applied, not just to S4.2, S4.3, and S5, but to all of extensions of the modal logic $\mathbf{S 4}$ covered in [29].

§2. Preliminaries. The logics we consider in this paper are all extensions of the commutative Full Lambek calculus. The grammar for formulas in this language ${ }^{1}$ is given below. Here Var is a countably infinite set of propositional variables.

$$
F:=p \in \operatorname{Var}|\top| \perp|1| 0|F \wedge F| F \vee F|F \cdot F| F \rightarrow F .
$$

We refer to the connective $\cdot$ as fusion (in the literature on linear logic, it is called either multiplicative conjunction or tensor). See [18] for an extensive discussion on the commutative Full Lambek calculus. Following the usual substructural convention, $\neg A$ abbreviates $A \rightarrow 0$.

Let $\operatorname{subf}(S)$ denote the set of subformulas in a formula/sequent $S$. Formulas are denoted by $A, B, C, \ldots$.

A multiset is a function mapping each element from a set (its 'universe') to a natural number (its 'multiplicity'). All multisets in this paper are finite in the sense that all but finitely many elements have multiplicity 0 .

Formula multisets (i.e., a multiset whose universe is the set of formulas) will be denoted by $\Gamma, \Delta, \ldots$. We say that ' $\Gamma$ contains $A$ ' to mean that the multiplicity of $A$ in $\Gamma$ is $\geq 1$. Also ' $\Gamma$ contains at most one formula' means that the multiplicity of some formula in $\Gamma$ is $\leq 1$ and every other formula has multiplicity 0 .

A sequent is a pair of formula multisets and is written $\Gamma \Rightarrow \Delta$. $\Gamma$ is called the antecedent and $\Delta$ the succedent. If $\Delta$ contains at most one formula then the sequent is said to be single-conclusioned, otherwise it is multi-conclusioned. The letter $\Pi$ is reserved to denote a multiset containing at most one formula.

2.1. Rule schemas, rule instances, and sequent calculus. A rule schema consists of some number of premise sequents and a single conclusion sequent, where the antecedent and succedent of each sequent may contain schematic-variables in addition to formulas. A rule schema with no premises is called an initial sequent.

An instance of the rule schema is obtained by the uniform instantiation of schematic-variables for concrete objects of the corresponding type, and the uniform

\footnotetext{
${ }^{1}$ We added the constants $\top$ (true) and $\perp$ (false) to the language. We are also able to conflate left and right implications to $\rightarrow$ since we are in the commutative setting.
} 
substitution of propositional variables (occurring in formulas) to formulas. It is typical in structural proof theory not to distinguish explicitly between a rule schema and its instance (indeed the word 'rule' is used for both), nor distinguish in notation between a schematic-variable and its instantiation. We follow this convention except where an explicit distinction is helpful; in that case an instance of the rule schema $r$ is denoted $\sigma(r)$ where $\sigma$ is a function that maps schematic-variables to concrete objects of the corresponding type. It will be helpful to permit $\sigma$ to be a map also from propositional variables to formulas.

EXAMPLE 1. Consider the following rule schemas.

$$
\Rightarrow(p \rightarrow q) \vee(q \rightarrow p) \quad(\operatorname{lin}) \quad \frac{\Gamma \Rightarrow A \quad A, \Delta \Rightarrow \Pi}{\Delta, \Gamma \Rightarrow \Pi} \text { (cut). }
$$

Above left, a function $\sigma$ mapping the propositional variables $p$ and $q$ to formulas yields a rule instance $\sigma($ lin $)$; e.g., $\sigma(p)=p \vee q$ and $\sigma(q)=q$ gives the rule instance with no premise and conclusion $\Rightarrow((p \vee q) \rightarrow q) \vee(q \rightarrow(p \vee q))$.

Above right, the rule schema is built from multiset schematic-variables $\Gamma, \Delta, \Pi$ and the formula schematic-variable $A$. For $\sigma(\Gamma)=\{p, p, q\} ; \sigma(\Delta)=\{r, p\} ; \sigma(\Pi)=\emptyset$; $\sigma(A)=r \wedge q$ we obtain the rule instance with premises $p, p, q \Rightarrow r \wedge q$ and $r \wedge q, r, p \Rightarrow$ and conclusion $r, p, p, p, q \Rightarrow$.

A rule schema is single-conclusioned if instantiations are restricted to singleconclusioned sequents. A sequent calculus is a finite set of rule schemas.

2.2. The sequent calculus $\mathbf{F L}_{\mathbf{e}}$ and its extensions. The sequent calculus $\mathbf{F L}_{\mathbf{e}}$ for the commutative Full Lambek calculus consists of the set of single-conclusioned rules schemas in Figure 1. We observe that no explicit exchange rule is needed for this calculus since sequents are built using multisets.

For a set $\mathcal{R}$ of rule schemas, the extension $\mathbf{F L}_{\mathbf{e} *}+\mathcal{R}$ is simply the set-union $\mathbf{F L}_{\mathbf{e} *} \cup \mathcal{R}$. For a set $\mathcal{A}$ of formulas, we write $\mathbf{F L}_{\mathbf{e} *}+\mathcal{A}$ for the extension of $\mathbf{F L}_{\mathbf{e} *}$ by the initial sequents $\Rightarrow A$ where $A \in \mathcal{A}$. Here are some well-known rule schemas: left weakening $\left(w_{l}\right)$, right weakening $\left(w_{r}\right)$, contraction $(c)$, and mingle [26] $(m)$

$$
\frac{\Delta \Rightarrow \Pi}{\Delta, A \Rightarrow \Pi}\left(w_{l}\right) \frac{\Delta \Rightarrow}{\Delta \Rightarrow \Pi}\left(w_{r}\right) \quad \frac{\Delta, A, A \Rightarrow \Pi}{\Delta, A \Rightarrow \Pi}(c) \frac{\Delta, \Gamma_{1} \Rightarrow \Pi \quad \Delta, \Gamma_{2} \Rightarrow \Pi}{\Delta, \Gamma_{1}, \Gamma_{2} \Rightarrow \Pi}(m)
$$

Rules schemas containing neither formulas nor propositional/formula schematicvariables will be called structural rules. We add a subscript to $\mathbf{F L}_{\mathbf{e}}$ to indicate an extension by one of the rules above, e.g., $\mathbf{F L}_{\mathbf{e c}}:=\mathbf{F L}_{\mathbf{e}}+\{(c)\}$. Also, $\mathbf{F L}_{\mathbf{e} *}\left(\mathbf{F L}_{\mathbf{e c} *}\right)$ denotes $\mathbf{F L}_{\mathbf{e}}\left(\mathbf{F} \mathbf{L}_{\mathbf{e c}}\right)$ extended by a combination of them. Finally, $\mathbf{F} \mathbf{L}_{\mathbf{e w}}$ is $\mathbf{F L}_{\mathbf{e w}_{\mathbf{l}} \mathbf{w}_{\mathbf{r}}}$.

2.3. (Cut-free) derivability and subformula property. Let $\mathcal{C}$ be any extension of FL $_{\mathbf{e}}$ by rule schemas, and $\mathcal{S}$ a set of sequents. A derivation (or proof) of a sequent $S$ $($ from $\mathcal{S})$ in $\mathcal{C}$ is defined in the usual way as a tree of sequents with root $S$ such that every internal node and its children correspond to the conclusion and premises of a rule instance of $\mathcal{C}$, and every leaf of the tree is an instance of an initial sequent from $\mathcal{C}$, or one of the sequents in $\mathcal{S}$.

A cut-free derivation does not contain any instance of the cut-rule. Let $\mathcal{S} \vdash_{\mathcal{C}} S$ $\left(\mathcal{S} \vdash_{\mathcal{C}}^{c f} S\right)$ denote that the sequent $S$ is derivable (cut-free derivable) from $\mathcal{S}$ in $\mathcal{C}$. 


$$
\begin{aligned}
& \overline{A \Rightarrow A}(\text { id }) \quad \frac{\Gamma \Rightarrow A \quad A, \Delta \Rightarrow \Pi}{\Gamma, \Delta \Rightarrow \Pi}(\text { cut }) \quad \frac{\Gamma \Rightarrow A_{i}}{\Gamma \Rightarrow A_{1} \vee A_{2}}\left(\vee_{R}\right)_{i \in\{1,2\}} \quad \frac{\Gamma, A \Rightarrow B}{\Gamma \Rightarrow A \rightarrow B}(\rightarrow R)
\end{aligned}
$$

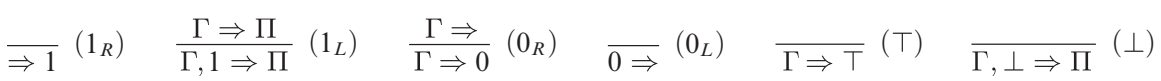

$$
\begin{aligned}
& \frac{\Gamma \Rightarrow A \quad \Delta \Rightarrow B}{\Gamma, \Delta \Rightarrow A \cdot B}\left(\cdot_{R}\right) \quad \frac{\Gamma, A \Rightarrow \Pi \quad \Gamma, B \Rightarrow \Pi}{\Gamma, A \vee B \Rightarrow \Pi}\left(\vee_{L}\right) \quad \frac{\Gamma, A, B \Rightarrow \Pi}{\Gamma, A \cdot B \Rightarrow \Pi}\left(\cdot_{L}\right) \\
& \frac{\Gamma \Rightarrow A \quad \Delta, B \Rightarrow \Pi}{\Gamma, \Delta, A \rightarrow B \Rightarrow \Pi}\left(\rightarrow_{L}\right) \quad \frac{\Gamma \Rightarrow A \quad \Gamma \Rightarrow B}{\Gamma \Rightarrow A \wedge B}\left(\wedge_{R}\right) \quad \frac{\Gamma, A_{i} \Rightarrow \Pi}{\Gamma, A_{1} \wedge A_{2} \Rightarrow \Pi}\left(\wedge_{L}\right)_{i \in\{1,2\}}
\end{aligned}
$$

Figure 1. The single-conclusioned sequent calculus $\mathbf{F L}_{\mathbf{e}}$.

If $\mathcal{S}$ is empty we write these as $\vdash_{\mathcal{C}} S$ and $\vdash_{\mathcal{C}}^{c f} S$ respectively. A derivation has the subformula property, and is called analytic, if all formulas occurring in it are subformulas of the endsequent.

We say that $\mathcal{C}$ is analytic (resp. $\mathcal{C}$ has cut-elimination) if every derivable sequent in $\mathcal{C}$ has an analytic derivation (resp. every derivation can be transformed into a cut-free derivation). It is well-known that $\mathbf{F L}_{\mathbf{e} *}$ has cut-elimination and is analyticits extensions by initial sequents are analytic only in trivial cases.

2.4. Logics and axiomatic extensions. A logic is a set of formulas closed under modus ponens (and necessitation, in the modal case) and the uniform substitution of propositional variables for arbitrary formulas.

For a logic $L$ and a finite set $\mathcal{A}$ of formulas, the axiomatic extension of $L$ by $\mathcal{A}$ (denoted $L+\mathcal{A}$ ) is the smallest logic containing $L$ and all formulas in $\mathcal{A}$.

Let $\mathcal{C}$ be any extension of $\mathbf{F L}_{\mathbf{e}}$ by rule schemas. We let $\operatorname{Thm}(\mathcal{C})$ denote the set of all formulas $F$ such that $\vdash_{\mathcal{C}} \Rightarrow F$. Since the cut-rule subsumes modus ponens, it is easily verified that $\operatorname{Thm}(\mathcal{C})$ is a logic and that $\operatorname{Thm}(\mathcal{C}+\mathcal{A})=\operatorname{Thm}(\mathcal{C})+\mathcal{A}$. This indicates that axiomatic extensions of the $\operatorname{logic} \operatorname{Thm}(\mathcal{C})$ are captured proof theoretically by the addition of initial sequent rule schemas to $\mathcal{C}$.

We say that $\mathcal{C}$ is a calculus for a logic $L$ if $\operatorname{Thm}(\mathcal{C})=L$.

Hypersequent calculi generalise sequent calculi by using a multiset of sequents (a hypersequent) rather than a single sequent for the premises and conclusion of the rule schemas. A hypersequent is written $S_{1}|\ldots| S_{n}$. Each $S_{i}$ (a sequent) is called a component.

Every sequent calculus $\mathbf{S}$ can be embedded into a hypersequent calculus HS: (i) replace each rule schema $(r)$ in $\mathbf{S}$ with $(\mathrm{Hr})$ (see below) where the hypersequent schematic-variable $G$ can be instantiated with a (possibly empty) hypersequent, and (ii) include the rules of external weakening (ew) and external contraction (ec) (the external exchange).

$$
\frac{S_{1} \quad \ldots \quad S_{n}}{S^{\prime}}(r) \quad \frac{G\left|S_{1} \quad \ldots \quad G\right| S_{n}}{G \mid S^{\prime}}(H r) \quad \frac{G}{G \mid S}(e w) \quad \frac{G|S| S}{G \mid S}(e c)
$$

It is easy to see that a sequent is derivable in $\mathbf{H S}$ iff it is derivable in $\mathbf{S}$. This observation will be used several times in this article. The additional expressivity of 
the hypersequent calculus comes from the use of rule schemas that act on multiple components of the conclusion simultaneously.

EXAmPLE 2. A sequent calculus for propositional Gödel logic extends $\mathbf{L J}=\mathbf{F L}_{\text {ecw }}$ by the initial sequent $\Rightarrow$ lin $($ lin $:=(p \rightarrow q) \vee(q \rightarrow p))$. The resulting system has neither cut-elimination nor analyticity. A hypersequent calculus with these properties can be obtained [3] by adding to HLJ the communication rule

$$
\frac{G\left|\Sigma_{1}, \Gamma_{1} \Rightarrow \Pi_{1} \quad G\right| \Sigma_{2}, \Gamma_{2} \Rightarrow \Pi_{2}}{G\left|\Sigma_{1}, \Gamma_{2} \Rightarrow \Pi_{1}\right| \Sigma_{2}, \Gamma_{1} \Rightarrow \Pi_{2}}(\mathrm{com}) .
$$

A cut-free hypersequent calculus for modal logic S5 with the subformula property is obtained by extending the hypersequent calculus HS4 for S4 with Avron's modalized splitting rule [4]:

$$
\frac{G \mid \Gamma_{1}, \square \Gamma_{2} \Rightarrow \square \Delta_{2}, \Delta_{1}}{G\left|\Gamma_{1} \Rightarrow \Delta_{1}\right| \square \Gamma_{2} \Rightarrow \square \Delta_{2}}\left(M S_{A v}\right) .
$$

In the above examples, the hypersequent schematic-variable $G$ is called the context . The remaining components are called the active components of the rule.

Notations and terminologies introduced for sequent calculi apply to hypersequent calculi in the obvious way.

§3. Twin perspectives. We now define bounded-axiomatisations and boundedanalytic sequent calculi.

A bounding function is any map $\psi$ which takes as arguments a set of formulas $\mathcal{A}$ (the axioms) and a formula $F$ (in practice, this is the formula that we wish to prove), and returns a set $\psi(\mathcal{A}, F)$ of instances of $\mathcal{A}$.

Definition 3 (Bounded-axiomatisation and bounded-axiomatisable). Let $\psi$ be a bounding function and $\mathcal{A}$ a finite set of formulas. A logic $L$ is said to be $\psi$-axiomatisation over $\operatorname{Thm}\left(\mathbf{F L}_{\mathbf{e} *}\right)$ w.r.t. $\mathcal{A}$ if $L=\operatorname{Thm}\left(\mathbf{F} \mathbf{L}_{\mathbf{e} *}\right)+\mathcal{A}$, and for every formula $F$ :

$$
F \in L \text { iff } \exists A_{1}, \ldots, A_{n} \in \psi(\mathcal{A}, F) \text { such that } A_{1} \cdot \ldots \cdot A_{n} \rightarrow F \in \operatorname{Thm}\left(\mathbf{F L}_{\mathbf{e} *}\right) .
$$

A logic $L$ is a $\psi$-axiomatisable over $\operatorname{Thm}\left(\mathbf{F L}_{\mathbf{e} *}\right)$ if it is a $\psi$-axiomatisation over $\operatorname{Thm}\left(\mathbf{F L}_{\mathbf{e} *}\right)$ w.r.t. some set $\mathcal{A}$.

Note that in the above definition, the same formula may appear multiple times in the list $A_{1}, \ldots, A_{n}$.

The definition of bounded-axiomatisation can be seen as a refinement of the local deduction theorem for commutative substructural logics. The latter can be formulated in our notation as:

TheOREm 4 [19]. Let $\mathcal{A}$ be a finite set of formulas and $L=\operatorname{Thm}\left(\mathbf{F L}_{\mathbf{e} *}\right)+\mathcal{A}$. Then for every formula $F$ :

$$
F \in L \text { iff } \exists A_{1}, \ldots, A_{n} \in \psi(\mathcal{A}) \text { such that }\left(A_{1} \wedge 1\right) \cdot \ldots \cdot\left(A_{n} \wedge 1\right) \rightarrow F \in \operatorname{Thm}\left(\mathbf{F L}_{\mathbf{e} *}\right),
$$

where $\psi(\mathcal{A})$ denotes the set of all instances of formulas in $\mathcal{A}$. 
The use of $\wedge 1$ in the local deduction theorem is inessential in our context, see the remark after Definition 7.

The key point is that Theorem 4 concerns a specific bounding function, i.e., the one that yields every instance of $\mathcal{A}$-Definition 3 parametrises this to some bounding function.

Obviously, it is preferable for a bounding function to be restrictive in the sense that the set $\psi(\mathcal{A}, F)$ is small. We shall see that finding a good bounding function for an axiomatic extension is nontrivial and depends on both the base logic and the choice of axioms. Below are four bounding functions - ordered from most restrictive to least - that will be the main focus of the present article.

1. The variable-bounding function $\psi_{v}(\mathcal{A}, F)$ contains all instances of formulas in $\mathcal{A}$ whose variables have been substituted by variables occurring in $F$.

2. The formula-bounding function $\psi_{f}(\mathcal{A}, F)$ contains all instances of formulas in $\mathcal{A}$ whose variables have been substituted by subformulas of $F$.

3. The set-bounding function $\psi_{s}(\mathcal{A}, F)$ contains all instances of formulas in $\mathcal{A}$ whose variables are substituted by non-repeating ${ }^{2}$ fusions of subformulas of $F$.

4. The multiset-bounding function $\psi_{m s}(\mathcal{A}, F)$ contains all instances of formulas in $\mathcal{A}$ whose variables have been substituted by fusions of subformulas of $F$.

In the definition of $\psi_{s}$ and $\psi_{m s}$ we admit empty fusions of subformulas, which are identified, as one might expect, with the constant 1.

Example 5. Let $\mathcal{A}=\{p \rightarrow 1\}$ and $F=r \wedge q$. Then

$-\psi_{v}(\mathcal{A}, F)=\{r \rightarrow 1, q \rightarrow 1\}$

$-\psi_{f}(\mathcal{A}, F)=\psi_{v}(\mathcal{A}, F) \cup\{r \wedge q \rightarrow 1\}$;

$-\psi_{s}(\mathcal{A}, F)=\psi_{f}(\mathcal{A}, F) \cup\{1 \rightarrow 1, r \cdot q \rightarrow 1,(r \wedge q) \cdot r \rightarrow 1,(r \wedge q) \cdot q \rightarrow 1\} ;$

$-\psi_{m s}(\mathcal{A}, F)=\psi_{s}(\mathcal{A}, F) \cup\{r \cdot r \rightarrow 1, q \cdot q \rightarrow 1, r \cdot r \cdot q \rightarrow 1, r \cdot q \cdot r \rightarrow 1, \ldots\}$.

Note that for finite $\mathcal{A}$, the substitution sets $\psi_{v}(\mathcal{A}, F), \psi_{f}(\mathcal{A}, F)$, and $\psi_{s}(\mathcal{A}, F)$ are finite, and $\psi_{m s}(\mathcal{A}, F)$ is infinite.

A $\psi$-axiomatisation has a natural proof theoretic analogue.

DeFinition 6 (Bounded-analytic sequent calculus). Let $\psi$ be a bounding function and $\mathcal{A}$ a finite set of formulas. A derivation of $\Rightarrow F$ in $\mathbf{F L}_{\mathbf{e} *}+\mathcal{A}$ is called $\psi$-analytic if every cut and every initial sequent instance from $\{\Rightarrow A \mid A \in \mathcal{A}\}$ appears in the context below with $A \in \psi(\mathcal{A}, F)$.

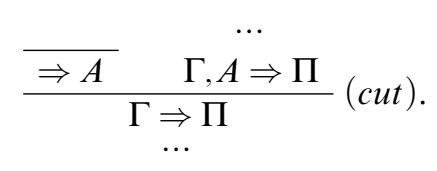

The sequent calculus $\mathbf{F L}_{\mathbf{e} *}+\mathcal{A}$ is $\psi$-analytic if every derivable formula has a $\psi$-analytic derivation.

Definition 7. A formula $A$ is weakenable over $\mathbf{F L}_{\mathbf{e} *}$ if $\vdash_{\mathbf{F L}_{\mathbf{e} *}} A \Rightarrow 1$.

\footnotetext{
${ }^{2}$ A fusion $A_{1} \cdot \ldots \cdot A_{n}$ is non-repeating if the $A_{i}$ 's are pairwise distinct.
} 
The weakenable requirement in the following is a very slight restriction, since for every set $\mathcal{A}$ of formulas: $\mathcal{A}_{\wedge 1}:=\{A \wedge 1 \mid A \in \mathcal{A}\}$ is a set of weakenable formulas in $\mathbf{F L}_{\mathbf{e} *}$, and $\operatorname{Thm}\left(\mathbf{F L}_{\mathbf{e} *}\right)+\mathcal{A}=\operatorname{Thm}\left(\mathbf{F L}_{\mathbf{e} *}\right)+\mathcal{A}_{\wedge 1}$. Of course, every formula is weakenable in the presence of the left weakening rule $\left(w_{l}\right)$.

Lemma 8. Let $\mathcal{A}$ be a set of weakenable formulas over $\mathbf{F L}_{\mathbf{e} *}$. The following are equivalent:

(i) The sequent calculus $\mathbf{F L}_{\mathbf{e} *}+\mathcal{A}$ is $\psi$-analytic.

(ii) The logic $\operatorname{Thm}\left(\mathbf{F L}_{\mathbf{e} *}\right)+\mathcal{A}$ is a $\psi$-axiomatisation over $\operatorname{Thm}\left(\mathbf{F L}_{\mathbf{e} *}\right)$ w.r.t. $\mathcal{A}$.

Proof. (i) to (ii): Suppose that $F \in \operatorname{Thm}\left(\mathbf{F L}_{\mathbf{e} *}\right)+\mathcal{A}=\operatorname{Thm}\left(\mathbf{F L}_{\mathbf{e} *}+\mathcal{A}\right)$. Since $\mathbf{F L}_{\mathrm{e} *}+\mathcal{A}$ is $\psi$-analytic by assumption, there is a $\psi$-analytic derivation of $\Rightarrow F$. Consider an occurrence of initial sequent instance of $\{\Rightarrow A \mid A \in \mathcal{A}\}$ in this derivation. By $\psi$-analyticity, we know that $A \in \psi(\mathcal{A}, F)$ and the occurrence of $\Rightarrow A$ is in a context as below on the left. Eliminate this initial sequent by replacing it with the proof figure below right, and propagate the additional formula $A$ downwards in the derivation.

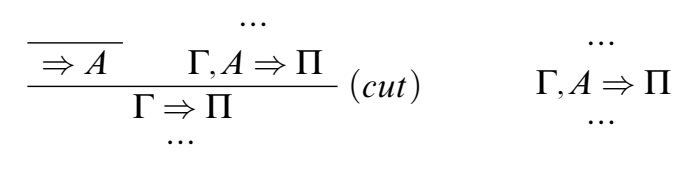

To propagate $A$ from the premises to the conclusion of a binary additive rule when $A$ only occurs in the antecedent of one premise, a copy needs to be supplied to the antecedent of the other premise. This can be achieved by making a cut on $A \Rightarrow 1$ like this, assuming that the premise is $\Sigma \Rightarrow \Delta$ :

$$
\frac{A \Rightarrow 1 \quad \frac{\Sigma \Rightarrow \Delta}{\Sigma, 1 \Rightarrow \Delta}}{\Sigma, A \Rightarrow \Delta}\left(1_{L}\right)
$$

By the assumption on weakenability, $A \Rightarrow 1$ is derivable in $\mathbf{F L}_{\mathbf{e} *}$. We obtain a proof of $A \Rightarrow F$ which has one less initial sequent from $\mathcal{A}$. Proceeding in this way, we eventually obtain a derivation of $A_{1}, \ldots, A_{n} \Rightarrow F$ without any initial sequents from $\mathcal{A}$, where each $A_{i} \in \psi(\mathcal{A}, F)$. It follows that $A_{1} \cdot \ldots \cdot A_{n} \rightarrow F \in \operatorname{Thm}\left(\mathbf{F L}_{\mathbf{e} *}\right)$.

(ii) to (i): Suppose that $\mathbf{F L}_{\mathrm{e} *}+\mathcal{A}$ derives $\Rightarrow F$. Then $F \in \operatorname{Thm}\left(\mathbf{F L}_{\mathbf{e} *}\right)+\mathcal{A}$, and by virtue of being a $\psi$-axiomatisation w.r.t. $\mathcal{A}$ there exists $A_{1}, \ldots, A_{n} \in \psi(\mathcal{A}, F)$ such that $A_{1} \cdot \ldots \cdot A_{n} \rightarrow F \in \operatorname{Thm}\left(\mathbf{F L}_{\mathbf{e} *}\right)$. By invertibility of the rules ${ }^{2} L$ and $\rightarrow_{R}$ there is a derivation of $A_{1}, \ldots, A_{n} \Rightarrow F$ in $\mathbf{F L}_{\mathbf{e} *}$. By cut-elimination in $\mathbf{F L}_{\mathbf{e} *}$, there is a cut-free derivation. By a cut on the latter with the initial sequent $\Rightarrow A_{1}$ we obtain $A_{2}, \ldots, A_{n} \Rightarrow F$. By a cut on the latter with $\Rightarrow A_{2}$ we obtain $A_{3}, \ldots, A_{n} \Rightarrow F$. Continuing in this way, we ultimately obtain a $\psi$-analytic derivation of $\Rightarrow F$ in $\mathbf{F L}_{\mathbf{e} *}+\mathcal{A}$.

An axiomatic extension over some base logic is called

- variable-axiomatisable if it is $\psi_{v}$-axiomatisable;

- formula-axiomatisable if it is $\psi_{f}$-axiomatisable;

- set-axiomatisable if it is $\psi_{s}$-axiomatisable;

- multiset-axiomatisable if it is $\psi_{m s}$-axiomatisable. 
An extension of a sequent calculus by initial sequents $\{\Rightarrow A \mid A \in \mathcal{A}\}$ is called

- variable-analytic if it is $\psi_{v}$-analytic;

- formula-analytic if it is $\psi_{f}$-analytic;

- set-analytic if it is $\psi_{s}$-analytic;

- multiset-analytic if it is $\psi_{m s}$-analytic.

3.1. Subformula property compared to $\psi$-analytic sequent calculus. For sequent calculus with the subformula property, every derivable sequent $\Rightarrow F$ has a derivation such that

every formula is a subformula of $F$.

Meanwhile in a $\psi$-analytic derivation of $\Rightarrow F$ in $\mathbf{F L}_{\mathbf{e} *}+\mathcal{A}$, every cut-formula and every initial sequent $\Rightarrow A$ belong to $\psi(\mathcal{A}, F)$. In such a derivation

every formula is a subformula of $F$ or of some $A \in \psi(\mathcal{A}, F)$.

Therefore, a $\psi$-analytic sequent calculus is a generalisation of a sequent calculus with the subformula property that preserves its original motivation: the restriction of the proof search space. This is especially the case when $\psi$ has a finite image. In the following sections we shall see that this generalisation is precisely what is needed to capture logics that do not satisfy the subformula property in the sequent calculus.

§4. Disjunction form and multiset-boundedness. To transform analytic hypersequent calculi into bounded-analytic sequent calculi we introduce the concept of a disjunction form of a hypersequent rule schema This is a disjunction of formulas that captures the essence of the rule.

We first present the formal definition of disjunction form, and show how to use it to obtain multiset-axiomatisations and multiset-analytic sequent calculi.

Although the definitions and results in this section and in the next one are formulated for commutative substructural logics, they adapt to other contexts, as demonstrated in Section 7 on modal logics.

\subsection{Disjunction form of a structural hypersequent rule.}

Convention. For a multiset $\mathcal{A}$ of formulas, let $\odot \mathcal{A}$ denote the fusion of all formulas in $\mathcal{A}$, and 1 if $\mathcal{A}$ is empty. When working in calculi which have weakening and contraction, we identify 1 and $\top, 0$ and $\perp$, as well as $\cdot$ and $\wedge$.

Definition 9. For a sequent $S=(\Gamma \Rightarrow \Pi)$, define $\Delta \# S:=(\Delta, \Gamma \Rightarrow \Pi)$.

The disjunction form is defined with respect to a rule schema (and not to a particular rule instance). Consequently, the disjunction form will represent all of its rule instances. Consider a structural hypersequent rule schema $(r)$

$$
\frac{G\left|T_{1} \quad \ldots \quad G\right| T_{l}}{G\left|S_{1}\right| \cdots \mid S_{n}}(r) .
$$

Here the $T_{i}$ 's are the active components of the premise, and the $S_{i}$ 's are the active components of the conclusion. Let $\left\{\Gamma_{i} \mid i \in I\right\}$ be the multiset schematicvariables from which the active components are built, and associate with each $\Gamma_{i}$ a 
propositional variable $\widehat{\Gamma}_{i}$. Given an instantiation $\sigma$ on $(r)$, we can then define the substitution $\widehat{\sigma}$ which maps each $\widehat{\Gamma}_{i}$ to the formula $\odot \sigma\left(\Gamma_{i}\right)$.

Definition 10. (Disjunction form of a rule) Let $(r)$ be given as above. The formula $A_{1} \vee \cdots \vee A_{n}$ built from the propositional variables $\widehat{\Gamma}_{i}(i \in I)$ is called a disjunction form of $(r)$ over $\mathbf{F L}_{\mathbf{e} *}$ if:

(splitting) For every rule instance $\sigma(r)$ and every $i \leq n$ :

$$
\left\{\sigma\left(T_{1}\right), \ldots, \sigma\left(T_{l}\right)\right\} \vdash_{\mathbf{F L}_{\mathbf{e} *}} \widehat{\sigma}\left(A_{i}\right) \# \sigma\left(S_{i}\right) .
$$

(provability) $\vdash_{\mathbf{H F L}_{\mathbf{e} *}+(r)} A_{1} \vee \cdots \vee A_{n}$.

(weakening) Each $A_{i}$ is weakenable over $\mathbf{F L}_{\mathbf{e} *}(\mathrm{cf}$. Definition 7).

We use the term "splitting" because the condition asserts that we can split the active components of a structural rule instance: the $i^{\text {th }}$ active component $\sigma\left(S_{i}\right)$ appended with the disjunct $\widehat{\sigma}\left(A_{i}\right)$ is cut-free derivable from the premises of the rule without using $(r)$. This can be depicted as follows:

$$
\frac{\sigma\left(T_{1}\right) \ldots \sigma\left(T_{l}\right)}{\sigma\left(S_{1}\right)|\cdots| \sigma\left(S_{n}\right)}(r) \leadsto\left\{\begin{array}{ccc}
\left\{\sigma\left(T_{1}\right), \ldots, \sigma\left(T_{l}\right)\right\} & & \left\{\sigma\left(T_{1}\right), \ldots, \sigma\left(T_{l}\right)\right\} \\
\vdots \mathbf{F L}_{\mathbf{e} *} & & \vdots \mathbf{F L}_{\mathbf{e} *} \\
\widehat{\sigma}\left(A_{1}\right) \# \sigma\left(S_{1}\right) & , \quad \ldots \quad, & \widehat{\sigma}\left(A_{1}\right) \# \sigma\left(S_{n}\right)
\end{array}\right\}
$$

By (provability), we assure that the disjunction form is not too strong, i.e., it must be a theorem of the logic under consideration.

ExAmple 11. $\left(\widehat{\Gamma}_{2} \rightarrow \widehat{\Gamma}_{1}\right) \vee\left(\widehat{\Gamma}_{1} \rightarrow \widehat{\Gamma}_{2}\right)$ is a disjunction form of

$$
\frac{G\left|\Sigma_{1}, \Gamma_{1} \Rightarrow \Pi_{1} \quad G\right| \Sigma_{2}, \Gamma_{2} \Rightarrow \Pi_{2}}{G\left|\Sigma_{1}, \Gamma_{2} \Rightarrow \Pi_{1}\right| \Sigma_{2}, \Gamma_{1} \Rightarrow \Pi_{2}}(\mathrm{com})
$$

over HLJ (cf. Example 2 and the case study in the introduction).

Lemma 12. Every disjunction form is weakenable over $\mathbf{F L}_{\mathbf{e} *}$.

Proof. Directly follows from the fact that all the disjuncts of a disjunction form are weakenable over $\mathbf{F L}_{\mathbf{e} *}$.

4.2. Multiset-axiomatisations/analyticity via the disjunction form. We are ready to prove the main result of this section.

THEOREM 13. Let $\mathbf{H F L}_{\mathbf{e} *}+\mathcal{R}$ be an extension of $\mathbf{H F L}_{\mathbf{e} *}$ by structural hypersequent rules and suppose that every rule in $\mathcal{R}$ has a disjunction form over $\mathbf{F L}_{\mathbf{e} *}$. Denote this set of disjunction forms as $\mathcal{A}$. Then

(i) $\operatorname{Thm}\left(\mathbf{H F L}_{\mathbf{e} *}+\mathcal{R}\right)=\operatorname{Thm}\left(\mathbf{F L}_{\mathbf{e} *}+\mathcal{A}\right)$,

(ii) $\mathbf{F L}_{\mathbf{e} *}+\mathcal{A}$ is a multiset-analytic sequent calculus, and

(iii) $\operatorname{Thm}\left(\mathbf{F L}_{\mathbf{e} *}\right)+\mathcal{A}$ is a multiset-axiomatisation over $\operatorname{Thm}\left(\mathbf{F L}_{\mathbf{e} *}\right)$ w.r.t. $\mathcal{A}$.

Proof. First observe that (ii) and (iii) are equivalent by Lemma 8.

To prove (i) and (iii) we make use of the following claim. 
$(\dagger)$ Every analytic $\mathbf{H F L}_{\mathbf{e} *}+\mathcal{R}$-derivation $\delta$ of $\Rightarrow F$ can be transformed into a $\mathbf{F L}_{\mathbf{e} *}$-derivation of $B_{1}, \ldots, B_{m} \Rightarrow F$ where $B_{i} \in \psi_{m s}(\mathcal{A}, F)$ for every $i \leq m$.

Indeed: $\operatorname{Thm}\left(\mathbf{H F L}_{\mathbf{e} *}+\mathcal{R}\right) \supseteq \operatorname{Thm}\left(\mathbf{F} \mathbf{L}_{\mathbf{e} *}+\mathcal{A}\right)$ by the (provability) property of disjunction forms, and the reverse inclusion $\operatorname{Thm}\left(\mathbf{H F L} \mathbf{L}_{\mathbf{e} *}+\mathcal{R}\right) \subseteq \operatorname{Thm}\left(\mathbf{F L}_{\mathbf{e} *}+\mathcal{A}\right)$ is a consequence of $(\dagger)$, so (i) follows.

Also, $\operatorname{Thm}\left(\mathbf{F L}_{\mathbf{e} *}\right)+\mathcal{A}=\operatorname{Thm}\left(\mathbf{F L}_{\mathbf{e} *}+\mathcal{A}\right)$ and this is $\operatorname{Thm}\left(\mathbf{H F L}_{\mathbf{e} *}+\mathcal{R}\right)$ by $(\mathrm{i})$. Therefore $F \in \operatorname{Thm}\left(\mathbf{F L}_{\mathbf{e} *}\right)+\mathcal{A}$ implies_using $(\dagger)$-that some $B_{1} \cdot \ldots \cdot B_{m} \rightarrow F \in$ $\operatorname{Thm}\left(\mathbf{F L}_{\mathbf{e} *}\right)$ with each $B_{i} \in \psi_{m s}(\mathcal{A}, F)$. So (iii) follows.

It remains to prove the claim $(\dagger)$.

We generalize the procedure illustrated in Section 1 for $\mathbf{H L J}+(\mathrm{com})$. To deal with derivations containing more than one application of $\mathcal{R}$-rules we first transform $\delta$ into an intermediary derivation of $B_{1}, \ldots, B_{m} \Rightarrow F$ in $\mathbf{H F L}_{\mathbf{e} *}$. Moreover, as the elimination of each $\mathcal{R}$-rule entails a duplication of parts of the derivation and hence might introduce new instances of rules in $\mathcal{R}$, we eliminate all lowermost $\mathcal{R}$-rules in $\delta$ simultaneously. In doing so, we ensure that after each reduction step, the maximal number of $\mathcal{R}$-instances on a branch in the proof-henceforth called the $\mathcal{R}$-rank of the derivation - decreases, and hence the whole procedure terminates.

Let $\sigma_{1}\left(r_{1}\right), \ldots, \sigma_{k}\left(r_{k}\right)$ the lowermost $\mathcal{R}$-instances in $\delta$. Assume $\sigma_{i}\left(r_{i}\right)$ is:

$$
\frac{\sigma_{i}(G)\left|\sigma_{i}\left(T_{1}\right) \quad \ldots \quad \sigma_{i}(G)\right| \sigma_{i}\left(T_{l_{i}}\right)}{\sigma_{i}(G)\left|\sigma_{i}\left(S_{1}\right)\right| \cdots \mid \sigma\left(S_{n_{i}}\right)} \sigma_{i}\left(r_{i}\right) .
$$

By assumption, there is a disjunction form $A_{i}=A_{1}^{i} \vee \cdots \vee A_{n_{i}}^{i} \in \mathcal{A}$ for $r_{i}$. Recall that $A_{i}$ is built from variables $\widehat{\Gamma}$ where $\Gamma$ is a multiset schematic-variable in the rule $r_{i}$, and that the substitution $\widehat{\sigma}_{i}$ is defined by setting $\widehat{\sigma}_{i}(\widehat{\Gamma}):=\odot \sigma_{i}(\Gamma)$.

From the subformula property of the derivation $\delta$ it follows that:

$(*)$ every instance of an $\mathcal{R}$-rule instantiates its multiset schematic-variables with a multiset of elements from $\operatorname{subf}(F)$,

and consequently, $\widehat{\sigma}_{i}\left(A_{i}\right) \in \psi_{m s}(\mathcal{A}, F)$. By the (splitting) property of the disjunction form we can eliminate the instance $\sigma_{i}\left(r_{i}\right)$ by introducing a formula $\widehat{\sigma}_{i}\left(A_{j}^{i}\right)$ to the antecedent of the $j$-active component in the conclusion $\left(j \leq n_{i}\right)$. This gives us $n_{i}$ ways of eliminating $\sigma_{i}\left(r_{i}\right)$.

Hence, in order to eliminate all lowermost instances $\sigma_{1}\left(r_{1}\right), \ldots, \sigma_{k}\left(r_{k}\right)$, we have to make $n_{1} \cdot \ldots \cdot n_{k}$ many choices. We may encode every such combined choice by a function in the set

$$
\Omega:=\left\{f:\{1, \ldots, k\} \rightarrow \mathbb{N} \mid \forall i\left(f(i) \leq n_{i}\right)\right\} .
$$

We fix one $f \in \Omega$ and formally describe a transformation $f(\delta)$ of the original proof $\delta$ which 'for all $i \leq k$ eliminate $\sigma_{i}\left(r_{i}\right)$ by adding $\widehat{\sigma}_{i}\left(A_{f(i)}^{i}\right)$ '. Indeed we simultaneously replace all instances $\sigma_{i}\left(r_{i}\right)$ (cf. (1) above) in $\delta$, for $i \leq k$, by

$$
\begin{gathered}
\sigma_{i}(G)\left|\sigma_{i}\left(T_{1}\right) \quad \ldots \quad \sigma_{i}(G)\right| \sigma_{i}\left(T_{l_{i}}\right) \\
\vdots \\
\frac{F_{i}(G) \mid \widehat{\sigma}_{i}\left(A_{f(i)}^{i}\right) \# \sigma_{i}\left(S_{f(i)}\right)}{\sigma_{i}(G)\left|\sigma_{i}\left(S_{1}\right)\right| \cdots\left|\widehat{\sigma}_{i}\left(A_{f(i)}^{i}\right) \# \sigma_{i}\left(S_{f(i)}\right)\right| \cdots \mid \sigma\left(S_{n_{i}}\right)}(e w) .
\end{gathered}
$$


Here, the dotted line indicates the $\mathbf{F L}_{\mathbf{e} *}$-derivation guaranteed by the (splitting) property; the side hypersequent $\sigma_{i}(G)$ can simply be appended to all sequents in this derivation. In words, the rule instance $\sigma_{i}\left(r_{i}\right)$ in $\delta$ has been replaced by a derivation (in $\mathbf{H F L}_{\mathbf{e} *}$ ) of the conclusion of $\sigma_{i}\left(r_{i}\right)$ from its premises where the formula $\widehat{\sigma}_{i}\left(A_{i, f(i)}\right)$ has been appended to the antecedent of the component $\sigma_{i}\left(S_{f(i)}\right)$. Next, the formula $\widehat{\sigma}_{i}\left(A_{i, f(i)}\right)$ is added to the corresponding antecedents of all hypersequents from the denoted (ew) down to the endsequent $\Rightarrow F$. When propagating $\widehat{\sigma}_{i}\left(A_{i, f(i)}\right)$ downwards from the premise to the conclusion of a binary additive rule, it is necessary to supply a copy of $\widehat{\sigma}_{i}\left(A_{i, f(i)}\right)$ to the antecedent of corresponding component in the other premise. This can be done using the rule $\left(w_{l}\right)$, if it is present in $\mathbf{F L}_{\mathbf{e} * *}$. Otherwise, the effect of $\left(w_{l}\right)$ can be simulated as follows, using the fact that $\widehat{\sigma}_{i}\left(A_{i, f(i)}\right)$ is weakenable (which is guaranteed by the (weakening) property of disjunction forms):

$$
\frac{\widehat{\sigma}_{i}\left(A_{i, f(i)}\right) \Rightarrow 1 \quad \frac{H \mid \Sigma \Rightarrow \Delta}{H \mid \Sigma, 1 \Rightarrow \Delta}}{H \mid \Sigma, \widehat{\sigma}_{i}\left(A_{i, f(i)}\right) \Rightarrow \Delta} 1_{L}(c u t) .
$$

Now having propagated all the $\widehat{\sigma}_{i}\left(A_{f(i)}^{i}\right)$ 's $(i \leq k)$ down to the endsequent, we obtain a derivation of

$$
\widehat{\sigma}_{1}\left(A_{f(1)}^{1}\right), \ldots, \widehat{\sigma}_{k}\left(A_{f(k)}^{k}\right) \Rightarrow F .
$$

Call this derivation $f(\delta)$, and note that its $\mathcal{R}$-rank is smaller than that of $\delta$. Recall that each disjunction form $A_{i}=A_{1}^{i} \vee \cdots \vee A_{n_{i}}^{i}$. To get the proof $\Omega(\delta)$ of

$$
\widehat{\sigma}_{1}\left(A_{1}\right), \ldots, \widehat{\sigma}_{k}\left(A_{k}\right) \Rightarrow F,
$$

we connect all the proofs $f(\delta)$ for every $f \in \Omega$ by repeatedly applying the $\left(\vee_{L}\right)$-rule to their conclusion. We already remarked that $\widehat{\sigma}_{i}\left(A_{i}\right) \in \psi_{m s}(\mathcal{A}, F)$. Furthermore, $\Omega(\delta)$ still satisfies $(*)$ because no new $\mathcal{R}$-instances have been introduced and every $\mathcal{R}$-instance of $\delta$ has either been eliminated or left unchanged. Lastly, the $\mathcal{R}$-rank of $\Omega(\delta)$ equals the maximal $\mathcal{R}$-rank of one of the $f(\delta)$ 's, and therefore is smaller than the $\mathcal{R}$-rank of $\delta$. It follows that we can repeat the above transformation to eventually obtain an $\mathcal{R}$-free derivation $\Omega^{*}(\delta)$ of

$$
B_{1}, \ldots, B_{m} \Rightarrow F \text { where each } B_{i} \in \psi_{m s}(\mathcal{A}, F) .
$$

Since this is a derivation in $\mathbf{H F L}_{\mathbf{e} *}$ and since this calculus contains no rule schemas that act on multiple components of the conclusion simultaneously, $\Omega^{*}(\delta)$ can be reduced to an $\mathbf{F L}_{\mathbf{e} \text { - }}$-proof by pruning components and branches. This concludes the proof of $(\dagger)$, and hence the proof of the theorem.

REMARK 14. Although the hypersequent calculi we consider have cut-elimination and the subformula property, the above proof argument relies only on the latter.

§5. Disjunction form for substructural logics. In the previous section the disjunction form was used to show that the corresponding sequent calculus is multiset- 
analytic. Here we show how to compute a disjunction form from the analytic hypersequent structural rules introduced in [12]. This leads to multiset-analytic sequent calculi for a large class of commutative substructural logics.

5.1. Analytic structural hypersequent rules. Analytic structural hypersequent rules (analytic rules, for short) are structural hypersequent rules that have exactly one active component in each premise and satisfy the following properties:

(linear conclusion) Every schematic-variable that occurs in the conclusion occurs exactly once there.

(separation) No multiset schematic-variable occurs in an antecedent position and in a succedent position.

(coupling) For each conclusion component with a multiset schematic-variable $\Pi$ as succedent, there is a multiset schematic-variable $\Sigma$ in the antecedent of the same component such that the pair $(\Sigma, \Pi)$ always occur together in the premises, and when $\Sigma$ occurs in a premise it occurs exactly once.

(subformula property) Each schematic-variable that occurs in the premise also occurs in the conclusion.

The addition of analytic rules to $\mathbf{H F L}_{\mathbf{e}}$ leads to cut-free hypersequent calculi with the subformula property for many substructural logics. These rules can be computed for a large class of axiomatic extensions of $\operatorname{Thm}\left(\mathbf{F L}_{\mathbf{e}}\right)$ in a uniform and systematic way. This result is based on the following syntactic classification of Hilbert axioms in the language of $\mathbf{F L}_{\mathbf{e}}$. Let $\mathcal{P}_{0}=\mathcal{N}_{0}$ be the set Var of propositional variables, and define

$$
\begin{aligned}
& \mathcal{P}_{n+1}:=1|\perp| \mathcal{N}_{n}\left|\mathcal{P}_{n+1} \vee \mathcal{P}_{n+}\right| \mathcal{P}_{n+1} \cdot \mathcal{P}_{n+1}, \\
& \mathcal{N}_{n+1}:=0|\top| \mathcal{P}_{n}\left|\mathcal{N}_{n+1} \wedge \mathcal{N}_{n+}\right| \mathcal{P}_{n+1} \rightarrow \mathcal{N}_{n+1}
\end{aligned}
$$

Observe that $U_{i} \subset V_{i+1}(U, V \in\{\mathcal{P}, \mathcal{N}\})$. For axiomatic extensions of $\mathbf{F L}_{\text {ew }}$ every formula in $\mathcal{P}_{3}$ can be transformed into analytic rules. In absence of weakening, only formulas in $\mathcal{P}_{3}^{\prime} \subset \mathcal{P}_{3}$ that are acyclic (i.e., those that do not lead the transformation into cycles) can be transformed into analytic rules. Here $\mathcal{P}_{3}^{\prime}$ is defined by the grammar $1|\perp| \mathcal{N}_{2} \wedge 1\left|\mathcal{P}_{3}^{\prime} \cdot \mathcal{P}_{3}^{\prime}\right| \mathcal{P}_{3}^{\prime} \vee \mathcal{P}_{3}^{\prime}$.

Definition 15 (Amenable). A set $\mathcal{A}$ of formulas is amenable if (i) $\mathcal{A} \subseteq \mathcal{P}_{3}$ and left weakening $p \cdot q \rightarrow p \in \mathcal{A}$, or (ii) $\mathcal{A} \subseteq \mathcal{P}_{3}^{\prime}$ consists of acyclic formulas.

A formula $A$ is amenable if $\{A\}$ is an amenable set.

THEOREM 16 [12]. (i) A finite set $\mathcal{R}_{\mathcal{A}}$ of analytic rules is computable from a finite set $\mathcal{A}$ of amenable formulas such that $\mathbf{H F L} \mathbf{L}_{\mathbf{e}}+\mathcal{R}_{\mathcal{A}}$ is a calculus for $\mathbf{F L}_{\mathbf{e}}+\mathcal{A}$ with cut-elimination and the subformula property.

(ii) Every analytic rule extension $\mathbf{H F L}_{\mathbf{e}}+\mathcal{R}$ is a calculus for an amenable axiomatic extension of $\mathbf{F} \mathbf{L}_{\mathbf{e}}$.

EXAmple 17. For the amenable set $\mathcal{A}=\{p \cdot q \rightarrow p$, lin $\} \subset \mathcal{P}_{3}$, the set $\mathcal{R}_{\mathcal{A}}$ that is computed using the algorithm in [12] consists of the analytic rules of weakening and (com) (see Example 2).

Meanwhile for the amenable set $\mathcal{A}^{\prime}=\{((p \rightarrow q) \wedge 1) \vee((q \rightarrow p) \wedge 1)\} \subset \mathcal{P}_{3}^{\prime}$, the set $\mathcal{R}_{\mathcal{A}^{\prime}}=\{(\mathrm{com})\}$. 
Theorem 16(i) in this paper is Theorem 5.6 in [12]. The proof in the latter relies on expressing every $\mathcal{P}_{3}^{\prime}$ axiomatic extension of $\mathbf{F L}_{\mathbf{e}}$ as an axiomatic extension by disjunctions of $\mathcal{N}_{2} \wedge 1$ formulas (see [12, Lemma 3.5]). The latter proof is incomplete as it does not cover cases like $\left(\left(\mathcal{P}_{3}^{\prime} \vee \mathcal{P}_{3}^{\prime}\right) \cdot\left(\mathcal{P}_{3}^{\prime} \vee \mathcal{P}_{3}^{\prime}\right)\right) \vee\left(\left(\mathcal{P}_{3}^{\prime} \vee \mathcal{P}_{3}^{\prime}\right) \cdot\left(\mathcal{P}_{3}^{\prime} \vee \mathcal{P}_{3}^{\prime}\right)\right)$. Nevertheless the result stands. An algebraic argument can be found in [13, Lemma 4.5]. Here is an alternative argument. By induction on the structure of $A$ we have $\mathbf{F L}_{\mathbf{e}} \vdash A \Rightarrow 1$, and thus $\mathbf{F L}_{\mathbf{e}} \vdash A, B \Rightarrow B$, for every $A, B \in \mathcal{P}_{3}^{\prime}$. Making use of the latter it can be seen that for every extension $L$ of $\mathbf{F L}_{\mathrm{e}}$ and $A, B, C \in \mathcal{P}_{3}^{\prime}$ : $\operatorname{Thm}(L+$ $A \cdot B)=\operatorname{Thm}(L+A+B)$ and $\operatorname{Thm}(L+(A \vee(B \cdot C)))=\operatorname{Thm}(L+A \vee B+A \vee C)$. Repeatedly apply the latter two equalities to express a $\mathcal{P}_{3}^{\prime}$ axiomatic extension as a disjunctions of $\mathcal{N}_{2} \wedge 1$ formulas.

REMARK 18. The amenable formulas define infinitely many substructural logics, including most of the $t$-norm and uninorm based logics [35], as well as the propositional interpolable intermediate logics (except $\mathrm{Bd}_{2}$ ). Moreover, as shown in [25], the hierarchy collapses at the next level in the sense that every formula is equivalent in $\mathbf{F L}_{\mathbf{e}}$ to some formula in $\mathcal{N}_{3}$.

5.2. Computing the disjunction form of an analytic rule. We now describe an algorithm which turns an analytic rule into a disjunction form. We occasionally write $A_{\wedge 1}$ for $A \wedge 1$ for brevity.

As the first step, select exactly one multiset schematic-variable occurrence in the active component of each premise ('distinguished variable occurrence') of the analytic rule. This induces an association of the distinguished variable (and the premise it is contained in) to the unique conclusion component containing this variable. For premises with a multiset schematic-variable $\Pi$ as succedent, we will choose as distinguished variable occurrence the multiset schematic-variable $\Sigma$ to which it is coupled (i.e., the coupling $(\Sigma, \Pi)$ in the definition of analytic rule).

The analytic rule together with the choice of distinguished variables can be pictured in association form (see Figure 2). Observe that:

- A multiset schematic-variable declared as distinguished in a premise whose active component has empty succedent may appear in a conclusion component with or without empty succedent.

- Distinct premises may be associated with the same conclusion component due to the same or different distinguished variables.

- Some conclusion components with empty succedent might not be associated with any premise (captured by the possibility that $s_{i}=0$ ).

- The $\mathcal{S}, \mathcal{T}$, and $\mathcal{U}$ multisets may contain further (non-distinguished) occurrences of the distinguished variables $\Gamma$ and $\Delta$, but no further occurrences of $\Sigma$ due to the coupling property. The multisets $\mathcal{V}$ and $\mathcal{W}$ do not contain any further occurrences of distinguished variables due to the linear conclusion property.

For a multiset $\mathcal{S}=\left\{\Gamma_{1}, \ldots, \Gamma_{n}\right\}$ of multiset schematic-variables, let $\widehat{\mathcal{S}}$ denote the multiset $\left\{\widehat{\Gamma}_{1}, \ldots, \widehat{\Gamma}_{n}\right\}$ of propositional variables.

Definition $19(\operatorname{Form}(r, i))$. For a rule $(r)$ in association form (Figure 2), let 
$\frac{\left\{G \mid \mathcal{S}_{i j}, \Sigma_{i} \Rightarrow \Pi_{i}\right\}_{i \in I, j \in J_{i}} \quad\left\{G \mid \mathcal{T}_{i j l}, \Gamma_{i j} \Rightarrow\right\}_{i \in I, j \leq r_{i}, l \in M_{i j}} \quad\left\{G \mid \mathcal{U}_{i j l}, \Delta_{i j} \Rightarrow\right\}_{i \in L, j \leq s_{i} l \in N_{i j}}}{G\left|\left[\mathcal{V}_{i}, \Gamma_{i 1}, \ldots, \Gamma_{i r_{i}}, \Sigma_{i} \Rightarrow \Pi_{i}\right]_{i \in I}\right|\left[\mathcal{W}_{i}, \Delta_{i 1}, \ldots, \Delta_{i s_{i}} \Rightarrow\right]_{i \in L}}$

FIGURE 2. Association form. $\mathcal{S}, \mathcal{T}, \mathcal{U}, \mathcal{V}, \mathcal{W}$ denote multisets of multiset schematicvariables. The distinguished variable occurrences in the premises and their associated occurrences in the components of the conclusion are indicated in boldface. The index sets $I, L, J_{i}, M_{i j}$, and $N_{i j}$ are assumed to be pairwise disjoint.

$$
\begin{array}{ll}
\operatorname{Form}(r, i):=\left(\odot \widehat{\mathcal{V}}_{i} \cdot \odot\left\{\widehat{\Gamma}_{i j} \wedge\left(\neg \bigvee_{l \in M_{i j}} \odot \widehat{\mathcal{T}}_{i j l}\right) \mid j \leq r_{i}\right\} \rightarrow \bigvee_{j \in J_{i}} \odot \widehat{\mathcal{S}}_{i j}\right)_{\wedge 1} \quad(i \in I), \\
\operatorname{Form}(r, i):=\left(\neg\left(\odot \widehat{\mathcal{W}}_{i} \cdot \odot\left\{\widehat{\Delta}_{i j} \wedge\left(\neg \bigvee_{l \in N_{i j}} \odot \widehat{\mathcal{U}}_{i j l}\right) \mid j \leq s_{i}\right\}\right)\right)_{\wedge 1} \quad(i \in L) .
\end{array}
$$

Finally, let $\operatorname{Form}(r):=\bigvee_{i \in I \cup L} \operatorname{Form}(r, i)$.

EXAMPLE 20. Here are association forms of three well-known structural rules. In (com), the choice of distinguished variables $\Sigma_{1}$ and $\Sigma_{2}$ is forced since the coupled variable in the antecedent is always chosen. In (lq), the $\Gamma$ could have been chosen as distinguished instead of $\Delta$. In (wc), the second occurrence of $\Delta$ could have been chosen as distinguished instead.

$$
\frac{G\left|\Gamma_{1}, \boldsymbol{\Sigma}_{\mathbf{1}} \Rightarrow \Pi_{1} \quad G\right| \Gamma_{2}, \boldsymbol{\Sigma}_{\mathbf{2}} \Rightarrow \Pi_{2}}{G\left|\Gamma_{2}, \boldsymbol{\Sigma}_{\mathbf{1}} \Rightarrow \Pi_{1}\right| \Gamma_{1}, \mathbf{\Sigma}_{\mathbf{2}} \Rightarrow \Pi_{2}}(\mathrm{com}) \quad \frac{G \mid \boldsymbol{\Delta}, \Gamma \Rightarrow}{G|\boldsymbol{\Delta} \Rightarrow| \Gamma \Rightarrow}(\text { lq }) \frac{G \mid \boldsymbol{\Delta}, \Delta \Rightarrow}{G \mid \boldsymbol{\Delta} \Rightarrow}(w c) \text {. }
$$

- Pattern-matching the (com) rule with Figure 2 we obtain:

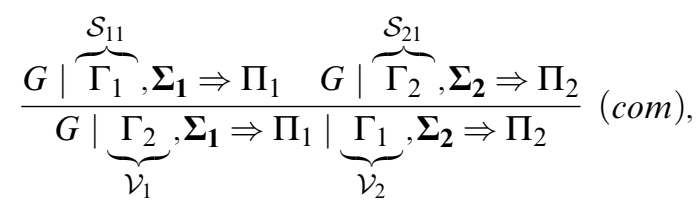

$I=\{1,2\}, L=\emptyset, \mathcal{V}_{1}=\left\{\Gamma_{2}\right\}, \mathcal{V}_{2}=\left\{\Gamma_{1}\right\}, J_{1}=J_{2}=\{1\}, \mathcal{S}_{11}=\left\{\Gamma_{1}\right\}, \mathcal{S}_{21}=\left\{\Gamma_{2}\right\}$, $r_{1}=r_{2}=0$, and therefore:

$\operatorname{Form}(\operatorname{com}, 1):=\left(\odot \widehat{\mathcal{V}}_{1} \cdot \odot \frac{\left.\left|\widehat{F}_{1 j} \lambda\left(V_{l \in M_{1 j}} \odot \widehat{\mathcal{T}}_{j l}\right)\right| j \leq T\right\}}{\longrightarrow} \rightarrow \bigvee_{j \in\{1\}} \odot \widehat{\mathcal{S}}_{1 j}\right)_{\wedge 1}$,

$\left.\operatorname{Form}(\operatorname{com}, 2):=\left(\odot \widehat{\mathcal{V}}_{2} \cdot \odot\left|\widehat{F}_{2 j} \wedge\left(V_{l \in M_{2 j}} \odot \widehat{\mathcal{F}}_{2 j l}\right)\right| j \leq r_{2}\right\} \rightarrow \bigvee_{j \in\{1\}} \odot \widehat{\mathcal{S}}_{2 j}\right)_{\wedge 1}$.

In the above, we have written $\widehat{\vartheta}$ to indicate that the term evaluates to 1 . (Recall that in $\mathbf{H F L}_{\mathbf{e} *}, \odot \mathcal{A}$ denotes the fusion of all formulas in $\mathcal{A}$, and 1 if $\mathcal{A}$ is empty.) So Form $(\mathrm{com})=\left(\widehat{\Gamma}_{2} \cdot 1 \rightarrow \widehat{\Gamma}_{1}\right)_{\wedge 1} \vee\left(\widehat{\Gamma}_{1} \cdot 1 \rightarrow \widehat{\Gamma}_{2}\right)_{\wedge 1}$.

- Pattern-matching the $(l q)$ rule with Figure 2 we obtain: 


$$
\frac{G|\Delta, \overbrace{\Gamma_{\Delta_{11}}}^{\Delta} \Rightarrow| \underbrace{\Gamma}_{\mathcal{W}_{2}} \Rightarrow}{G \mid \mathcal{U}_{111}}(l q),
$$

$I=\emptyset, L=\{1,2\}, s_{1}=1, s_{2}=0, \mathcal{N}_{11}=\{1\}, \mathcal{W}_{1}=\emptyset, \mathcal{W}_{2}=\mathcal{U}_{111}=\Gamma, \Delta_{11}=\Delta$, and therefore:

$$
\begin{aligned}
& \left.\operatorname{Form}(l q, 1):=\left(\neg\left(\odot \widehat{\widehat{U}_{1 j}} \wedge\left(\neg \bigvee_{l \in N_{1 j}} \odot \widehat{\mathcal{U}}_{1 j l}\right) \mid j \leq 1\right\}\right)\right)_{\wedge 1}, \\
& \left.\left.\operatorname{Form}(l q, 2):=\left(\neg\left(\odot \widehat{\mathcal{W}}_{2} \cdot \odot \frac{\left\{\widehat{\Delta}_{2 j}\right.}{\mid V_{l \in N_{2 j}}\left(\widehat{u t}_{2 j l}\right)}\right) ; \leq 2\right\}\right)\right)_{\wedge 1} .
\end{aligned}
$$

So Form $(l q)=(\neg(1 \cdot(\widehat{\Delta} \wedge \neg \widehat{\Gamma})))_{\wedge 1} \vee(\neg(\widehat{\Gamma} \cdot 1))_{\wedge 1}$.

- Similarly, for $(w c)$ one obtains Form $(w c)=(\neg(1 \cdot(\widehat{\Delta} \wedge \neg \widehat{\Delta})))_{\wedge 1}$.

THeOREM 21. Form $(r)$ is a disjunction form of the analytic rule $(r)$ over $\mathbf{H F L}_{\mathbf{e} *}$.

Proof. Given an analytic rule $(r)$, obtain Form $(r)$ from its association form. We require (cf. Definition 10) (i) provability, i.e., $\vdash_{\mathbf{H F L}_{\mathbf{e}}+(r)} \Rightarrow$ Form(r), and (ii) splitting. Weakening is satisfied by construction.

(i) First repeatedly apply to $\Rightarrow \operatorname{Form}(r)$ the rules $(e c)$ and $\left(\vee_{R}\right)$ to obtain the hypersequent $\Rightarrow \operatorname{Form}(r, i)|\cdots| \Rightarrow \operatorname{Form}(r, l)$, for all $i \in I$ and $l \in L$. Then apply the invertible rules $\left(\vee_{L}\right),\left(\rightarrow_{R}\right),\left({ }_{L}\right)$ backwards from each component to get the hypersequent below. The instantiation $\sigma$ making this hypersequent the conclusion of an instance $\sigma(r)$ of $(r)$ in association form (cf. Figure 2) is obtained by patternmatching (refer to variables shown above the hypersequents),

$$
\begin{aligned}
& {[\widehat{\mathcal{V}}_{i},\{\overbrace{\widehat{\Gamma}_{i j} \wedge\left(\neg \bigvee_{l \in M_{i j}} \odot \widehat{T}_{i j l}\right)}^{\Gamma_{i j}} \mid j \leq r_{i}\} \Rightarrow \overbrace{\bigvee_{j \in J_{i}}^{\Sigma_{i} \odot \widehat{\mathcal{S}}_{i j}}}^{\Pi_{i}}]_{i \in I} \mid} \\
& \times\left[\widehat{\mathcal{W}}_{i},\{\overbrace{\widehat{\Delta}_{i j} \wedge\left(\neg \bigvee_{l \in N_{i j}} \odot \widehat{\mathcal{U}}_{i j l}\right)}^{\Delta_{i j}} \mid j \leq s_{i}\} \Rightarrow\right]_{i \in L} .
\end{aligned}
$$

- For $i \in I: \quad \sigma(G):=\emptyset \quad \sigma\left(\Sigma_{i}\right):=\emptyset \quad \sigma\left(\Pi_{i}\right):=\bigvee_{j \in J_{i}} \odot\left(\widehat{\mathcal{S}}_{i j}\right)$,

for every $\mathcal{V}_{i}=\left\{Q_{1}, \ldots, Q_{n}\right\}$, set $\sigma\left(Q_{s}\right):=\widehat{Q}_{s}$,

finally, set $\sigma\left(\boldsymbol{\Gamma}_{i j}\right):=\widehat{\boldsymbol{\Gamma}}_{i j} \wedge \neg \bigvee_{l \in M_{i j}} \odot\left(\widehat{\mathcal{T}}_{i j l}\right) \quad\left(j \leq r_{i}, l \in M_{i j}\right)$.

- For $i \in L$ : for every $\mathcal{W}_{i}=\left\{Q_{1}, \ldots, Q_{n}\right\}$, set $\sigma\left(Q_{s}\right):=\widehat{Q}_{s}$,

finally, set $\sigma\left(\boldsymbol{\Delta}_{i j}\right):=\widehat{\boldsymbol{\Delta}}_{i j} \wedge \neg \bigvee_{l \in N_{i j}} \odot\left(\widehat{\mathcal{S}}_{i j l}\right) \quad\left(j \leq s_{i}, l \in N_{i j}\right)$. 
After applying $\sigma(r)$ backwards to the hypersequent above, it suffices to derive each premise of $\sigma(r)$ in $\mathbf{H F L}_{\mathbf{e}}$.

We illustrate with the premise $G \mid \mathcal{S}_{i j}, \boldsymbol{\Sigma}_{i} \Rightarrow \Pi_{i}$ of $(r)\left(i \in I, j \in J_{i}\right)$. In $\sigma(r)$, since $\sigma\left(\Sigma_{i}\right)=\emptyset=\sigma(G)$ this becomes $\sigma\left(\mathcal{S}_{i j}\right) \Rightarrow \bigvee_{j^{\prime} \in J_{i}} \odot\left(\widehat{\mathcal{S}}_{i j^{\prime}}\right)$. Obtain $\sigma\left(\mathcal{S}_{i j}\right) \Rightarrow$ $\odot\left(\overrightarrow{\widehat{\mathcal{S}}}_{i j}\right)$ using $\left(\vee_{R}\right)$. Let $\overrightarrow{\mathcal{S}}_{i j}=\left\{P_{1}, \ldots, P_{n}\right\}$ (each $P_{s}$ is a multiset schematic-variable). Applying $\left(\cdot{ }_{R}\right)$ backwards to the latter sequent we obtain $\sigma\left(P_{s}\right) \Rightarrow \widehat{P}_{s}(1 \leq s \leq n)$. It remains to verify derivability of the latter. Since $P_{s}$ occurs in the premise in the antecedent, it must occur in the conclusion (subformula property) in the antecedent (separation). Additionally it cannot be a $\Sigma$ variable since coupled variables occur only as indicated in Figure 2 by the (coupling) condition. Therefore either $P_{s} \in \mathcal{V}_{i}$, $P_{s} \in \overrightarrow{\mathcal{W}}_{i}, P_{s}=\Gamma_{u v}$, or $P_{s}=\Delta_{u v}$. In the first two cases, due to the definition of $\sigma\left(\overrightarrow{\mathcal{V}}_{i}\right)$ and $\sigma\left(\overrightarrow{\mathcal{W}}_{i}\right)$, we have the assignment $\sigma\left(P_{s}\right):=\widehat{P}_{s}$ and hence derivability. In the latter two cases we get $\widehat{\boldsymbol{\Gamma}}_{u v} \wedge \neg \bigvee_{l \in M_{u v}} \odot\left(\widehat{\mathcal{T}}_{u v l}\right) \Rightarrow \widehat{\Gamma}_{u v}$ and $\widehat{\boldsymbol{\Delta}}_{u v} \wedge \neg \bigvee_{l \in N_{u v}} \odot\left(\widehat{\mathcal{S}}_{u v l}\right) \Rightarrow \widehat{\Delta}_{u v}$ respectively. Applying $\left(\wedge_{L}\right)$ backwards we get $\widehat{\Gamma}_{u v} \Rightarrow \widehat{\Gamma}_{u v}$ and $\widehat{\Delta}_{u v} \Rightarrow \widehat{\Delta}_{u v}$.

(ii) Proving that $\operatorname{Form}(r)$ satisfies (splitting) follows from a straightforward inspection so we simply set out what needs to be proved. Let $(r)$ be given as

$$
\frac{\mathcal{H}}{G \mid\left[S_{i}\right]_{i \in I \cup L}}(r) .
$$

We have to show that for any instantiation $\sigma$ and $i \in I \cup L, \sigma\left(\widehat{\sigma}(\operatorname{Form}(r, i)) \# S_{i}\right)$ is derivable from the active components of the premises in $\sigma(\mathcal{H})$ in $\mathbf{F L}_{\mathbf{e} *}$. We illustrate the case $i \in I$. The sequent $\sigma\left(\widehat{\sigma}(\operatorname{Form}(r, i)) \# S_{i}\right)$ is

$$
\widehat{\sigma}(\operatorname{Form}(r, i)), \sigma\left(\mathcal{V}_{i}\right), \sigma\left(\Gamma_{i 1}\right), \ldots, \sigma\left(\Gamma_{i r_{i}}\right), \sigma\left(\Sigma_{i}\right) \Rightarrow \sigma\left(\Pi_{i}\right) .
$$

From Definition 19 we have that $\widehat{\sigma}(\operatorname{Form}(r, i))$ has the following form:

$$
\left(\odot \sigma\left(\mathcal{V}_{i}\right) \cdot \odot\left\{\sigma\left(\Gamma_{i j}\right) \wedge\left(\neg \bigvee_{l \in M_{i j}} \odot \sigma\left(\overrightarrow{\mathcal{T}}_{i j l}\right)\right) \mid j \leq r_{i}\right\} \rightarrow \bigvee_{j \in J_{i}} \odot \sigma\left(\mathcal{S}_{i j}\right)\right)_{\wedge 1}
$$

Applying the logical rules backwards to suitably decompose the formula $\widehat{\sigma}(\operatorname{Form}(r, i))$, one obtains a proof of (2) from the active components of $(r)$.

REMARK 22. An alternative way of obtaining a disjunction formula is by reversing the algorithm in [12]. The resulting formula would be different, although equivalent, to the one we compute. For example, in absence of weakening, for the (com) rule we would get the formula $\left(\widehat{\Gamma}_{2} \rightarrow \widehat{\Gamma}_{1}\right)_{\wedge 1} \vee\left(\widehat{\Gamma}_{1} \rightarrow \widehat{\Gamma}_{2}\right)_{\wedge 1}$ which is slightly simpler than Form $(\mathrm{com})$ in Example 20. The reason for not taking the reversing approach is that the method given here works uniformly for substructural and modal logics, and it does not require any familiarity with the algorithm in [12].

\subsection{Multiset-axiomatisations/analyticity for substructural logics.}

THEOREM 23. Let $\mathcal{A}$ be an amenable set. Then:

1. There is a set $\mathcal{A}^{\prime}$ computable from $\mathcal{A}$ such that $\operatorname{Thm}\left(\mathbf{F} \mathbf{L}_{\mathbf{e} *}+\mathcal{A}^{\prime}\right)=\operatorname{Thm}\left(\mathbf{F} \mathbf{L}_{\mathbf{e} *}+\right.$ A) and $\mathbf{F L}_{\mathbf{e} *}+\mathcal{A}^{\prime}$ is a multiset-analytic sequent calculus.

2. $\operatorname{Thm}\left(\mathbf{F L}_{\mathbf{e} *}+\mathcal{A}\right)$ is multiset-axiomatisable over $\operatorname{Thm}\left(\mathbf{F L}_{\mathbf{e} *}\right)$. 
Proof. By Theorem 16, we can compute from $\mathcal{A}$ a set $\mathcal{R}_{A}$ of hypersequent rules such that $\operatorname{Thm}\left(\mathbf{F L}_{\mathbf{e} *}+\mathcal{A}\right)=\operatorname{Thm}\left(\mathbf{H F L}_{\mathbf{e} *}+\mathcal{R}_{A}\right)$ and $\mathbf{H F L}_{\mathbf{e} *}+\mathcal{R}_{A}$ is analytic. By Definition 19 and Theorem 21, we can compute from the set $\mathcal{R}_{A}$ of rules a set $\mathcal{A}^{\prime}$ of disjunction forms. Then from Theorem 13 we have that $\mathbf{F} \mathbf{L}_{\mathbf{e} *}+\mathcal{A}^{\prime}$ is a multisetanalytic sequent calculus, and that $\operatorname{Thm}\left(\mathbf{F L}_{\mathbf{e} *}+\mathcal{A}^{\prime}\right)$ is a multiset-axiomatisation over $\operatorname{Thm}\left(\mathbf{F L}_{\mathbf{e} *}\right)$ w.r.t. $\mathcal{A}^{\prime}$. To conclude the proof, it suffices to observe that $\operatorname{Thm}\left(\mathbf{F L}_{\mathbf{e} *}+\right.$ $\left.\mathcal{A}^{\prime}\right)=\operatorname{Thm}\left(\mathbf{H F L}_{\mathbf{e} *}+\mathcal{R}_{A}\right)=\operatorname{Thm}\left(\mathbf{F L}_{\mathbf{e} *}+\mathcal{A}\right)$.

§6. Stronger forms of boundedness. In the last section we identified a large class of substructural logics that are multiset-axiomatisable. We now consider sufficient conditions for strengthening this result to set- and formula-axiomatisable, and study its consequences. We also study the properties of variable-axiomatisations, which appeared as the 'simple substitution property' in the literature.

The stronger forms of axiomatisable rely on the presence of certain structural rules, notably contraction. Formula-axiomatisations (resp. set-axiomatisations) lead to a PTIME (resp. EXPTIME) embedding. An EXPSPACE upper bound then follows for every amenable intermediate logic. It turns out that contraction and mingle suffice for set-axiomatisable. We then obtain a 2EXPTIME upper bound for amenable extensions of $\mathbf{F L}_{\mathbf{e c m} *}$. To the best of our knowledge, a bound applying to all these extensions is new. Finally we discuss how variable-axiomatisations can be exploited to obtain interpolation.

6.1. Set-axiomatisations. Multiset-axiomatisations imply set-axiomatisations whenever the structural rules of contraction and mingle (or weakening) are present.

Lemma 24. Let $\mathbf{F L}_{\mathbf{e c m} *}+\mathcal{A}$ be multiset-axiomatisable over $\mathbf{F L}_{\mathbf{e c m} *}$. Then $\mathbf{F L}_{\mathbf{e c m} *}+$ $\mathcal{A}$ is a set-axiomatisable over $\mathbf{F L}_{\mathbf{e c m} *}$.

Proof. $\quad \mathbf{F L}_{\text {ecm } *}$ establishes $A \leftrightarrow A \cdot A$, and hence also its contextualized version $B(A) \leftrightarrow B(A \cdot A)$. From this it follows that for any sets of axioms $\mathcal{A}$ and formula $F$, the sets $\mathcal{A}\left[\psi_{m s}(F)\right]$ and $\mathcal{A}\left[\psi_{s}(F)\right]$ are equivalent over $\mathbf{F} \mathbf{L}_{\mathbf{e c m} *}$.

Corollary 25. Every amenable extension $\mathbf{F L}_{\mathbf{e c m} *}+\mathcal{A}$ is set-axiomatisable over $\mathbf{F L}_{\mathrm{ecm} *}$.

Proof. From Theorem 23 and Lemma 24.

An embedding of a logic $L$ into a logic $K$ is a function $\rho$ on formulas such that $F \in L \leftrightarrow \rho(F) \in K$. The embedding is PTIME (EXPTIME) if the function is computable in PTIME (EXPTIME).

LEMMA 26. 1. If $\mathbf{F L}_{\mathbf{e c} *}+\mathcal{A}$ is a set-axiomatisable over $\mathbf{F L}_{\mathbf{e} *}$ then there is an EXPTIME embedding from $\operatorname{Thm}\left(\mathbf{F L}_{\mathbf{e c} *}+\mathcal{A}\right)$ into $\operatorname{Thm}\left(\mathbf{F L}_{\mathbf{e c} *}\right)$.

2. If $\mathbf{F L}_{\mathbf{e c} *}+\mathcal{A}$ is a formula-axiomatisable over $\mathbf{F L}_{\mathbf{e} *}$ then there is a PTIME embedding from $\operatorname{Thm}\left(\mathbf{F L}_{\mathbf{e c} *}+\mathcal{A}\right)$ into $\operatorname{Thm}\left(\mathbf{F L}_{\mathbf{e c} *}\right)$.

Proof. 1. By the definition of set-axiomatisable, $F \in \operatorname{Thm}\left(\mathbf{F L}_{\mathbf{e c} *}+\mathcal{A}\right)$ iff

$$
\exists A_{1}, \ldots, A_{n} \in \psi_{s}(\mathcal{A}, F) \text { such that } \vdash_{\mathbf{F L}_{\mathbf{e c} *}} A_{1}, \ldots, A_{n} \Rightarrow F \text {. }
$$

Since $\psi_{s}(\mathcal{A}, F)$ is a finite set, we have that $\left(^{*}\right)$ is equivalent to the following (contraction is needed in the left-to-right direction of the equivalence since a formula 
$A \in \psi_{s}(\mathcal{A}, F)$ might appear multiple times in the list $\left.A_{1}, \ldots, A_{n}\right)$.

$$
\vdash_{\mathbf{F L}_{\mathbf{e c} *}} \odot\left\{A \wedge 1 \mid A \in \psi_{s}(\mathcal{A}, F)\right\} \Rightarrow F .
$$

Therefore the following is an embedding of $\operatorname{Thm}\left(\mathbf{F L}_{\mathbf{e c} *}+\mathcal{A}\right)$ into $\operatorname{Thm}\left(\mathbf{F L}_{\mathbf{e c} *}\right)$.

$$
F \mapsto \odot\left\{A \wedge 1 \mid A \in \psi_{s}(\mathcal{A}, F)\right\} \rightarrow F .
$$

The time to compute values of this function is polynomial in the size of the set $\psi_{s}(\mathcal{A}, F)$ and that is exponential in $F$.

2. Arguing as in 1., we see that the following function is an embedding of $\operatorname{Thm}\left(\mathbf{F L}_{\mathbf{e c} *}+\mathcal{A}\right)$ into $\operatorname{Thm}\left(\mathbf{F L}_{\mathbf{e c} *}\right)$.

$$
F \mapsto \odot\left\{A \wedge 1 \mid A \in \psi_{f}(\mathcal{A}, F)\right\} \rightarrow F .
$$

It is computable in PTIME because the size of $\psi_{f}(\mathcal{A}, F)$ is polynomial in $F$.

We obtain the following uniform decidability and complexity upper bound.

COROllary 27. Derivability in amenable extensions of $\mathbf{F L}_{\mathbf{e c m} *}$ is 2EXPTIME.

Proof. By Theorem 23 and Lemmas 24 and 26, derivability for every amenable extension of $\mathbf{F L}_{\mathbf{e c m}}$ can be reduced to derivability in $\mathbf{F L}_{\mathbf{e c m}}$, in EXPTIME. By Theorem 29 below we have that $\operatorname{Thm}\left(\mathbf{F L}_{\mathbf{e c m}}\right)$ is itself in EXPTIME. The 2EXPTIME upper bound follows.

EXAMPLE 28. Our decidability and complexity results apply to a large class of logics including Uninorm Mingle Logic UML [34] axiomatized as $\mathbf{F L}_{\mathbf{e c m}}+(p \rightarrow$ $q)_{\wedge 1} \vee(q \rightarrow p)_{\wedge 1}$ (see [33] for a proof of decidability), as well as the substructural $\operatorname{logics} \mathbf{F} \mathbf{L}_{\mathbf{e c m}}+((p \cdot \neg p) \rightarrow p)_{\wedge 1}$, and $\mathbf{F} \mathbf{L}_{\mathbf{e c m}}+(B w k)(k \geq 2)$. Here $(B w k)$ is the wellknown formula $\vee_{i=0}^{k}\left(p_{i} \rightarrow \vee_{j \neq i} p_{j}\right)_{\wedge 1}$ whose addition to intuitionistic logic yields the logic of bounded width intuitionistic Kripke models.

In terms of the algebraic semantics [18], the corollary above applies to the equational theory of the corresponding classes of residuated lattices.

A 2EXPTIME upper bound for $\mathbf{F L}_{\mathbf{e c m}}$ already appears in [44]. The following improves on this bound. A PSPACE lower bound for this logic was given in [21].

TheOREM 29. Derivability in $\mathbf{F L}_{\mathrm{ecm}}$ is EXPTIME.

ProOF. Since $\vdash_{\text {FLecm }_{\text {ecm }}}^{c f} \Gamma \Rightarrow A$ iff $\vdash_{\text {FLecm }_{\text {ecm }}}^{c f} \Rightarrow \odot \Gamma \rightarrow A$, it suffices to decide derivability of a formula. Let $\mathbf{F L}_{\mathbf{e c m}}^{\mathbf{s}}$ be the calculus whose rule figures are the same as those of $\mathbf{F L}_{\mathbf{e c m}}$, but whose sequents have the form $X \Rightarrow A$ where $X$ is a set of formulas (instead of a multiset as in $\mathbf{F L}_{\mathbf{e c m}}$ ). Since $\vdash_{\mathbf{F L}_{\mathrm{ecm}}}^{c f} A \leftrightarrow A \cdot A$, the claim below follows from a standard induction on the height of the derivation.

$$
\text { For every formula } A: \vdash_{\mathbf{F L}_{\text {ecm }}}^{c f} \Rightarrow A \text { iff } \vdash_{\mathbf{F L}_{\text {ecm }}^{\mathrm{s}}}^{c f} \Rightarrow A \text {. }
$$

It suffices therefore to search for a proof of $\Rightarrow A$ in $\mathbf{F L}_{\mathbf{e c m}}^{\mathbf{s}}$. In a cut-free derivation in $\mathbf{F L}_{\text {ecm }}^{\mathbf{s}}$ of $\Rightarrow A$, each antecedent is a subset of $\operatorname{subf}(A)$. Since $|\operatorname{subf}(A)| \leq|A|$, there are at most $2^{|A|}$ such subsets, and at most $|A|$ possibilities for the succedent. There are thus at most $K:=2^{|A|} \cdot|A|$ sequents that can occur in a cut-free proof of $\Rightarrow A$. 
Using forward proof search, we can decide in exponential time ${ }^{3}$ if $\Rightarrow A$ is derivable as follows.

First, write down on a tape of the Turing Machine, each of the $K$ sequents that can occur in the proof (this takes time polynomial in $K$ ). Mark each initial sequent in this list as proved. Now consider the following algorithm:

1. For each triple of sequents $\left(S_{1}, S_{2}, S_{3}\right)$ such that $S_{1}$ and $S_{2}$ are marked proved and $S_{3}$ is unmarked, if there is a binary rule instance in $\mathbf{F L}_{\mathbf{e c m}}^{\mathbf{s}}$ with premises $S_{1}$ and $S_{2}$ and conclusion $S_{3}$, then mark $S_{3}$ as newly proved. Proceed similarly with the unary rules of $\mathbf{F L}_{\mathrm{ecm}}^{\mathbf{s}}$ using a tuple.

2. If none of the sequents is marked newly proved after Step 1 then terminate. Otherwise, replace every newly proved mark with proved, and go to Step 1.

It is easily verified that $\Rightarrow A$ is derivable iff $\Rightarrow A$ is marked proved at termination. Step 1 takes time polynomial in $K$ for each triple, and there are at most $K^{3}$ different triples ( $K^{2}$ different tuples). At each iteration, by Step 2 , the algorithm terminates unless the number of sequents marked proved increases. Hence the algorithm must terminate after at most $K$ iterations. It follows that the algorithm has a runtime that is polynomial in $K$, and thus exponential in $|A|$.

6.2. Formula and variable boundedness. The previous subsection made use of contraction and mingle to obtain set-axiomatisations. Replacing mingle with weakening, and hence considering the $\mathbf{L J}$ calculus for intuitionistic logic, opens the door to even sharper results. Here we present two sufficient conditions for a multiset-axiomatisation of $\mathbf{L} \mathbf{J}$ to imply a formula-axiomatisation. Adding a further condition implies a variable-axiomatisation and regains the sufficient condition for the simple substitution property presented in [23]. Recall that $A \wedge 1 \leftrightarrow A$ holds in the presence of weakening, and hence all references to the constant 1 are omitted in this section.

Let $A(B / p)$ denote the formula obtained from $A$ by substituting every occurrence of the propositional variable $p$ in $A$ with the formula $B$.

Definition 30 ( $\Omega$-propagation property). Formula $A$ has the $\Omega$-propagation property for a set $\Omega$ of binary connectives if for variables $p, q, r$ and every $\diamond \in C$,

$$
\vdash_{\mathbf{L J}} A(q / p), A(r / p) \Rightarrow A(q \diamond r / p) .
$$

Note that this condition is trivial for variables $p$ not occurring in $A$.

LEMMA 31. Let $\mathbf{L J}+\mathcal{A}$ be a multiset-axiomatisation over $\mathbf{L J}$ w.r.t. $\mathcal{A}$. It is a formula-axiomatisation if every $A \in \mathcal{A}$ has the $\{\wedge\}$-propagation property.

Proof. Suppose that $\vdash_{\mathbf{L J}+\mathcal{A}} F$. Since $\mathbf{L J}+\mathcal{A}$ is a multiset-axiomatisation, there exist $A_{1}, \ldots, A_{n}$ such that $\vdash_{\mathbf{L J}} A_{1}, \ldots, A_{n} \Rightarrow F$ and each $A_{i}$ is a multisetsubstitution, i.e., a substitution of the propositional variables of some formula in $\mathcal{A}$ by conjunctions of subformulas of $F$. By repeatedly using the $\{\wedge\}$-propagation property, some sequent $\vdash_{\mathbf{L J}} A_{1}^{\prime}, \ldots, A_{m}^{\prime} \Rightarrow F$ is derivable such that each $A_{i}^{\prime}$ is a substitution of the propositional variables of some formula in $\mathcal{A}$ by subformulas of $F$, i.e., a formula-substitution.

\footnotetext{
${ }^{3}$ We thank B. Lellmann for bringing this argument to our attention.
} 
Determining if an axiom has the $\{\wedge\}$-propagation property can be tedious, e.g., try checking this property for the $B c_{k}$ axiom (Kripke models with $k$ worlds) $p_{0} \vee$ $\left(p_{0} \rightarrow p_{1}\right) \vee \cdots \vee\left(p_{0} \wedge \cdots \wedge p_{k-1} \rightarrow p_{k}\right)$.

We now introduce an inductive criterion to simplify this check.

In the following, let $p \in$ Var be an arbitrary propositional variable, and let $A(B)$ abbreviate $A(B / p)$. Let $U_{p}$ denote the set of formulas that possess the $\{\wedge\}$ propagation property with respect to the variable $p$ :

$$
U_{p}=\left\{A \mid \forall q, r \in \text { Var. } \vdash_{\mathbf{L J}} A(q), A(r) \Rightarrow A(q \wedge r)\right\} .
$$

The following is immediate.

Lemma 32. If $A \in U_{p}$ for every variable $p$ occurring in $A$, then $A$ has the $\{\wedge\}$ propagation property.

Recall that an occurrence of a propositional variable $p$ is negative (positive) in a formula $A$ if it occurs on the left hand side of an odd (resp. even) number of implications. Define the following sets of formulas:

$$
\begin{aligned}
& U_{p}^{*}=\left\{A \mid \forall q, r \in \text { Var. } \vdash_{\mathbf{L J}} A(q) \Rightarrow A(q \wedge r)\right\} ; \\
& D_{p}=\left\{A \mid \forall q, r \in \text { Var. } \vdash_{\mathbf{L J}} A(q \wedge r) \Rightarrow A(q) \& \vdash_{\mathbf{L J}} A(q \wedge r) \Rightarrow A(r)\right\} ; \\
& N_{p}=\{A \mid \text { poccurs only negatively in } A\} .
\end{aligned}
$$

As a mnemonic, $U$ stands for ' u pwards propagation' (going from simple instances up to conjunctive instances), $U^{*}$ for 'strong upwards propagation', $D$ stands for ‘d ownwards propagation' (going from conjunctive instances down to simple instances), and of course $N$ stands for n egative. For sets $M, N$ of formulas and a binary connective $\diamond$, define $M \diamond N$ to be the set $\{A \diamond B \mid A \in M, B \in N\}$.

LEMMA 33. The following holds.

$1 p \in U_{p}$;

2 If $p$ does not occur in $A$, then $A \in U_{p}^{*} \cap U_{p} \cap D_{p}$;

$3 N_{p} \subseteq U_{p}^{*}$;

$4 D_{p} \rightarrow U_{p} \subseteq U_{p}$;

$5 U_{p}^{*} \vee U_{p} \subseteq U_{p}$.

Proof. (1) Since $\vdash_{\text {LJ }} q, r \Rightarrow q \wedge r$. (2) holds trivially. (3) Let $A \in N_{p}$, and let $\delta$ be the standard proof of $A(q) \Rightarrow A(q)$. Start constructing a proof $\delta^{\prime}$ of $A(q) \Rightarrow A(q \wedge r)$ bottom up by imitating the proof steps in $\delta$. Whenever the formula $q \wedge r$ appears isolated in $\delta^{\prime}$, then this is on the left hand side of a sequent because $A \in N_{p}$. We can thus apply a cut with $q \wedge r \Rightarrow q$ to (again, reading the proof bottom up) replace $q \wedge r$ with $q$. Then copy the remaining steps of $\delta$. (4) Let $A \in D_{p}$ and $B \in U_{p}$, and consider the following derivation showing that $A \rightarrow B \in U_{p}$ :

$$
\begin{array}{ccc} 
& \text { since } A \in D_{p} & \text { since } B \in U_{p} \\
\text { since } A \in D_{p} & \frac{A(q \wedge r) \Rightarrow A(r)}{B(q), B(r) \Rightarrow B(q \wedge r)} \\
\frac{A(q \wedge r) \Rightarrow A(q)}{B(q),(A \rightarrow B)(r), A(q \wedge r) \Rightarrow B(q \wedge r)} & \frac{(A \rightarrow B)(q),(A \rightarrow B)(r), A(q \wedge r) \Rightarrow B(q \wedge r)}{(A \rightarrow B)(q),(A \rightarrow B)(r) \Rightarrow(A \rightarrow B)(q \wedge r)}
\end{array}
$$


(5) Let $A \in U_{p}^{*}$ and $B \in U_{p}$. Start constructing a proof of $(A \vee B)(q),(A \vee B)(r) \Rightarrow$ $(A \vee B)(q \wedge r)$ by applying the rule $\left(\vee_{L}\right)$ twice. We then have to check provability of the following four sequents:

(i) $A(q), A(r) \Rightarrow(A \vee B)(q \wedge r)$;

(ii) $A(q), B(r) \Rightarrow(A \vee B)(q \wedge r)$;

(iii) $B(q), A(r) \Rightarrow(A \vee B)(q \wedge r)$;

(iv) $B(q), B(r) \Rightarrow(A \vee B)(q \wedge r)$.

Now (i)-(iii) are provable since $A \in U_{p}^{*}$. Also (iv) is provable since $B \in U_{p}$.

Together, Lemmas 32 and 33 provide a convenient sufficient condition for the $\{\wedge\}$-propagation property. Here are two examples.

ExAmPle 34. The Bck axiom $p_{0} \vee\left(p_{0} \rightarrow p_{1}\right) \vee \cdots \vee\left(p_{0} \wedge \cdots \wedge p_{k-1} \rightarrow p_{k}\right)$ enjoys the $\{\wedge\}$-propagation property. Indeed by Lemma 33(1) $p_{0}$ is in $U_{p_{0}}$; by Lemma 33(3) the remaining disjuncts are in $U_{p_{0}}^{*}$ and hence by Lemma 33(5) the axiom is in $U_{p_{0}}$. Next observe that by Lemma $33(1,2,4) p_{0} \rightarrow p_{1}$ is in $U_{p_{1}}$; by Lemma 33(2,3) the remaining disjuncts are in $U_{p_{0}}^{*}$ and hence by Lemma 33(5) the axiom is in $U_{p_{1}}$. Continuing in this way we can argue that the axiom is in $U_{p_{i}}$ for all $i \leq k$ and hence enjoys the $\{\wedge\}$-propagation property.

ExAmple 35. Consider the linearity axiom $A=(p \rightarrow q) \vee(q \rightarrow p)$. To show that $A \in U_{p}$, we observe that $(p \rightarrow q) \in U_{p}^{*}$ by Lemma 33(3) and $(q \rightarrow p) \in U_{p}$ by Lemmas $33(1,2,4)$. So $A \in U_{p}$ by Lemma 33(5). By a symmetric argument, $A \in U_{q}$. So by Lemma $32,(p \rightarrow q) \vee(q \rightarrow p)$ has the $\{\wedge\}$-propagation property and by Lemma 31 we obtain that the standard axiomatisation for Gödel logic $\mathbf{L} \mathbf{J}+(p \rightarrow q) \vee(q \rightarrow p)$ is a formula-axiomatisation.

6.3. Variable-axiomatisations. Variable-axiomatisations in intermediate logics were investigated in [23, 40-42] as the simple substitution property.

It is important to note that this is a property of a specific axiomatisation rather than a property of the logic, i.e., alternative axiomatisations of the same logic may not have the property. For example, the standard axiomatisation $\mathbf{L J}+(p \rightarrow$ $q) \vee(q \rightarrow p)$ for Gödel logic is not a variable-axiomatisation. The formula $\neg p \vee \neg \neg p$ is a counterexample since it is a theorem of Gödel logic and $\mathbf{L J}$ does not prove $(p \rightarrow p) \vee(p \rightarrow p) \Rightarrow \neg p \vee \neg \neg p$. As shown in [40], a variable-axiomatisation of Gödel logic is $\mathbf{L J}+(p \rightarrow q) \vee((p \rightarrow q) \rightarrow p)+(\neg p \vee \neg \neg p)$.

A simple sufficient criterion for a variable-axiomatisation is presented in [23] using a propagation property. We reformulate that proof in our setting.

Lemma 36. An arbitrary extension $\mathbf{L} \mathbf{J}+\mathcal{A}$ is a variable-axiomatisation w.r.t. $\mathcal{A}$ if every $A \in \mathcal{A}$ has the $\{\wedge, \vee, \rightarrow\}$-propagation property.

Proof. If $\vdash_{\mathbf{L J}+\mathcal{A}}(\Rightarrow F)$ for some formula $F$, then $\vdash_{\mathbf{L J}} A_{1}, \ldots, A_{n} \Rightarrow F$ for some instances $A_{i}$ of axioms in $\mathcal{A}$. By repeatedly applying the $\{\wedge, \vee, \rightarrow\}$-propagation property of the axioms, we can replace the list $A_{1}, \ldots, A_{n}$ of assumptions by a list $A_{1}^{\prime}, \ldots, A_{m}^{\prime}$ of variable instances (i.e., the variables of the axiom substituted by variables). If any variable occurring in some $A_{i}^{\prime}$ does not occur in $F$, we can uniformly rename such a variable with one that does occur in $F$. We obtain a proof of $A_{1}^{\prime \prime}, \ldots, A_{m}^{\prime \prime} \Rightarrow F$ where each $A_{i}^{\prime \prime} \in \mathcal{A}\left[\psi_{v}(F)\right]$. In this way we witness that $\mathbf{L J}+\mathcal{A}$ is a variable-axiomatisation w.r.t. $\mathcal{A}$. 
Observe that Lemma 36 applies to arbitrary extensions of $\mathbf{L J}$ but Lemma 31 requires a multiset-axiomatisation. This is because an arbitrary formula instance $A^{\prime}$ of $\mathcal{A}$ cannot be transformed to an element of $\mathcal{A}\left[\psi_{f}(F)\right]$ by variable renaming. In contrast, an arbitrary variable instance $A^{\prime}$ of $\mathcal{A}$ is transformable to an element in $\mathcal{A}\left[\psi_{v}(F)\right]$ via variable renaming. Lemma 36 is used in [23] to show that classical logic $\mathbf{L} \mathbf{J}+(\neg p \vee p)$ and $\mathbf{L Q}=\mathbf{L J}+(\neg p \vee \neg \neg p)$ are variable-axiomatisations.

A logic $L$ has the Craig interpolation property if $A \rightarrow B \in L$ implies the existence of a formula $I$ with $(A \rightarrow I) \wedge(I \rightarrow B) \in L$ and $\operatorname{Var}(I) \subseteq \operatorname{Var}(A) \cap \operatorname{Var}(B)$. We reproduce below the elegant argument from [40] where the Craig interpolation property for $\mathbf{L Q}$ is induced from $\mathbf{L J}$ by using a variable-axiomatisation. An analogous argument applies to classical logic.

THeOREM 37. LQ has the Craig interpolation property.

Proof. For a formula $X$, let $A_{X}$ denote the conjunction of all formulas $\neg q \vee \neg \neg q$ such that $q \in \operatorname{Var}(X)$. If $\vdash_{\mathbf{L Q}} B \rightarrow C$, then since $\mathbf{L Q}$ is a variable-axiomatisation over LJ w.r.t. $(\neg p \vee \neg \neg p)$ we know $\vdash_{\mathbf{L J}} A_{B \rightarrow C} \rightarrow(B \rightarrow C)$. Since $\neg q \vee \neg \neg q$ has only one variable, every conjunct in $A_{B} \wedge A_{C}$ appears in $A_{B \rightarrow C}$ and vice versa. Thus $\vdash_{\mathbf{L J}} A_{B \rightarrow C} \leftrightarrow A_{B} \wedge A_{C}$, and hence $\vdash_{\mathbf{L J}}\left(A_{B} \wedge A_{C}\right) \rightarrow(B \rightarrow C)$. It follows that $\vdash_{\mathbf{L J}}$ $\left(A_{B} \wedge B\right) \rightarrow\left(A_{C} \rightarrow C\right)$. By the interpolation property of $\mathbf{L} \mathbf{J}$, there is a formula $I$ such that $\operatorname{Var}(I) \subseteq \operatorname{Var}(A) \cap \operatorname{Var}(B)$ and $\vdash_{\mathbf{L J}}\left(\left(A_{B} \wedge B\right) \rightarrow I\right) \wedge\left(I \rightarrow\left(A_{C} \rightarrow C\right)\right.$. Then the latter formula is also provable in $\mathbf{L} \mathbf{Q}$. Since $A_{B}, A_{C} \in \mathbf{L Q}$, by rearranging and some cuts $\vdash_{\mathbf{L Q}}(B \rightarrow I) \wedge(I \rightarrow C)$. Hence $I$ is a Craig interpolant of $A \rightarrow B$ in $\mathbf{L Q}$.

In [41] it is shown that classical logic and LQ are the only consistent variableaxiomatisable logics over $\mathbf{L J}$ that are axiomatisable by a single-variable formula. The same paper shows that all finite-valued Gödel logics are variable-axiomatisable over LJ. We remark that these results have been generalised in [42] using algebraic methods to establish necessary and sufficient criterion for variable-axiomatisable intermediate logics on a finite slice (see [22]).

§7. Methodology extended to modal logics. In this section we demonstrate how the methodology applies to other non-classical logics, taking here the example of modal logics. In Section 7.1 we establish formula-axiomatisations for the case study of S4.2. Next we apply our methodology to the uniform hypersequent calculi in [29] for a large class of modal logics extending $\mathbf{S} \mathbf{4}$ by Lahav's simple frame properties. Finally, in Section 7.3 we sharpen the technique even further to obtain a new syntactic proof of a well-known result: the sequent calculus for $\mathbf{S 5}$ is analytic.

7.1. Formula-axiomatisation of S4.2. Throughout, a formula is called boxed if it has the form $\square A$.

The modal logic $\mathbf{S 4}$ of pre-ordered (reflexive and transitive) Kripke frames is obtained by the addition of the following two rules to the standard multi-conclusion sequent calculus $\mathbf{L K}$ for classical propositional logic.

$$
\frac{A, \Gamma \Rightarrow \Delta}{\square A, \Gamma \Rightarrow \Delta}(T) \quad \frac{\square \Gamma \Rightarrow A}{\square \Gamma \Rightarrow \square A}(4) .
$$

The modal logic S4.2 extends S4 by the axiom $\diamond \square p \rightarrow \square \diamond p$. This logic is characterised semantically as the logic of directed pre-ordered Kripke frames. A 
cut-free hypersequent calculus HS4.2 for $\mathbf{S 4 . 2}$ is obtained (see [28]) by lifting S4 to a hypersequent calculus HS4 and then adding the modal rule

$$
\frac{G \mid \square \Gamma, \square \Delta \Rightarrow}{G|\square \Gamma \Rightarrow| \square \Delta \Rightarrow}(R M S) .
$$

Paralleling the argument in Section 4.2, we now transform cut-free HS4.2-proofs into bounded analytic sequent calculus proofs. First, using the rules $(e c)$ and $\left(w_{l}\right)$ it is easy to see that any instance of $(R M S)$ can be replaced by multiple applications of its single formula variant

$$
\frac{G \mid \square A, \square \Delta \Rightarrow}{G|\square A \Rightarrow| \square \Delta \Rightarrow}\left(R M S_{1}\right) .
$$

As a disjunction form for $\left(R M S_{1}\right)$, we choose the formula $\square \neg \square \square p \vee \square \neg \square \neg \square p$ (the formal computation of the modal disjunction form is explained in Section 7.2). This is equivalent to the axiom instance $\diamond \square(\square p) \rightarrow \square \diamond(\square p)$ and hence the provability property is satisfied. The following two derivations show the splitting property for $\sigma(p)=A$ :

$$
\begin{array}{cc}
\frac{\square A \Rightarrow \square A}{\square A \Rightarrow \square \square A} & \frac{\square \Delta, \square A \Rightarrow}{\square \Delta \Rightarrow \neg \square A} \\
\frac{\square A, \neg \square \square A \Rightarrow}{\square} & (4) \\
\frac{\square, \square \neg \square \square A \Rightarrow}{\square}(T) & \frac{\square \Delta \Rightarrow \square \neg \square A}{\square \Delta, \neg \square \neg \square A \Rightarrow}
\end{array}(T)
$$

We can then eliminate applications of $\left(R M S_{1}\right)$ in proofs of $\Rightarrow F$ by adding the formula $\square \neg \square \square A$ or the formula $\square \neg \square \neg \square A$ into the corresponding antecedent of the rule conclusion. We then propagate these formulas downwards in each of the two derivations. Crucially, since both formulas are boxed, adding them to the antecedent of applications of (4) yields a legal instance of (4).

Finally, combine the two derivations by a disjunction introduction to obtain a proof of $\square \neg \square \square A \vee \square \neg \square \neg \square A \Rightarrow F$. As $\square A$ appeared in the cut-free hypersequent calculus proof, it must be a subformula of $F$. Eliminate all instances of $\left(R M S_{1}\right)$ and reduce the resulting $\mathbf{H S 4}$ proof into a sequent calculus proof.

TheOREM 38. The logic $\mathbf{S 4 . 2}$ is formula-axiomatisable over $\mathbf{S 4}$. In other words, this means $F \in \mathbf{S 4 . 2}$ iff there are boxed subformulas $A_{1}, \ldots, A_{n}$ of $F$ such that $\bigwedge_{i=1}^{n}\left(\square \neg \square A_{i} \vee \square \neg \square \neg A_{i}\right) \rightarrow F \in \mathbf{S 4}$.

We obtain formula-axiomatisations - as opposed to set-axiomatisationsbecause we replaced $\square \Gamma$ in $(R M S)$ with $\square A$ in $\left(R M S_{1}\right)$.

7.2. Bounded-analytic sequent calculi for simple frame properties. Boundedanalytic sequent calculi for modal logics - extending the methodology for substructural logics - can be obtained from a uniform presentation of the hypersequent rules. We illustrate using the rules in [29] for extensions of $\mathbf{S 4}$.

Let $n$ be a positive natural number and $S=\left\{\left(S_{R}^{i}, S_{=}^{i}\right)\right\}_{i \in I}$ for some finite index set $I$ such that every $S_{R}^{i}$ and $S_{=}^{i}$ is a subset of $\{1, \ldots, n\}$ and every $S_{R}^{i} \cup S_{=}^{i}$ is non-empty. This is what is called a "normal description of an $n$-simple $\mathcal{L}_{1}$-formula" [29, Definition 4]. Any such description defines a first-order formula 


$$
\forall w_{1} \ldots \forall w_{n} \exists u \bigvee_{i \in I}\left(\bigwedge_{i \in S_{R}^{i}} w_{i} R u \wedge \bigwedge_{i \in S_{\underline{\underline{i}}}} w_{i}=u\right) .
$$

This gives rise to the class of Kripke frames satisfying this formula. The modal logic of $S$ is defined as the set of formulas valid on this class of frames. For each such logic, an analytic hypersequent calculus is obtained in [29] by computing the following hypersequent rule "induced by $S$ for transitive modal logics"

$$
\frac{G \mid \cup_{j \in S_{R}^{i}} \otimes \Gamma_{j}^{\prime}, \cup_{j \in S_{\underline{i}}^{i}} \Sigma_{j} \Rightarrow \cup_{j \in S_{\underline{i}}^{i}} \Pi_{j} \quad(i \in I)}{G\left|\square \Gamma_{1}^{\prime}, \Sigma_{1} \Rightarrow \Pi_{1}\right| \cdots \mid \square \Gamma_{n}^{\prime}, \Sigma_{n} \Rightarrow \Pi_{n}}
$$

$\square \Gamma_{i}$ and $\nabla \Gamma_{i}$ are standard notation for multiset schematic-variables that are instantiable by sets $\left\{\square A_{1}, \ldots, \square A_{k}\right\}$ and $\left\{A_{1}, \square A_{1}, \ldots, A_{k}, \square A_{k}\right\}$ respectively.

EXAMPLE 39.

- Set $S_{\text {lin }}=\left\{\left(S_{R}^{i}, S_{=}^{i}\right)\right\}_{i \in\{1,2\}}$ with $S_{R}^{1}=\{1\}, S_{=}^{1}=\{2\}, S_{R}^{2}=\{2\}$, and $S_{=}^{2}=\{1\}$. The modal logic of $S_{\text {lin }}$ is the set of modal formulas valid on the class of frames satisfying $\forall w_{1} w_{2} \exists u .\left(w_{1} R u \wedge w_{2}=u\right) \vee\left(w_{2} R u \wedge w_{1}=u\right)$. This defines the analytic rule

$$
\frac{G\left|\otimes \Gamma_{1}^{\prime}, \Sigma_{2} \Rightarrow \Pi_{2} \quad G\right| \nabla \Gamma_{2}^{\prime}, \Sigma_{1} \Rightarrow \Pi_{1}}{G\left|\square \Gamma_{1}^{\prime}, \Sigma_{1} \Rightarrow \Pi_{1}\right| \square \Gamma_{2}^{\prime}, \Sigma_{2} \Rightarrow \Pi_{2}} r\left(S_{\text {lin }}\right) .
$$

Therefore HS4 $+r\left(S_{\text {lin }}\right)$ is a cut-free hypersequent calculus for $S_{l i n}$.

- For $S=\{(\{i, j\}, \emptyset) \mid 1 \leq i<j \leq n\}$, the corresponding first-order formula is $\forall w_{1} \ldots \forall w_{n} \exists u \bigvee_{1<i<j<n}\left(w_{i}=u \wedge w_{j}=u\right)$ (bounded cardinality) and the hypersequent rule is

$$
\frac{\left\{G \mid \Sigma_{i}, \Sigma_{j} \Rightarrow \Pi_{i}, \Pi_{j}\right\}_{1 \leq i<j \leq n}}{G\left|\square \Gamma_{1}^{\prime}, \Sigma_{1} \Rightarrow \Pi_{1}\right| \cdots \mid \square \Gamma_{n}^{\prime}, \Sigma_{1} \Rightarrow \Pi_{n}} r(S) .
$$

We shall obtain the following analogue of Theorem 13 for the function Form ${ }^{\square}$ defined below that maps an analytic hypersequent rule to a modal formula.

THEOREM 40. For every normal description $S$ and the analytic hypersequent rules $\mathcal{R}$ induced by it, the modal logic $\mathrm{Thm}(\mathbf{H S} \mathbf{4}+\mathcal{R})$ is a set-axiomatisation over $\mathbf{S} 4$ w.r.t. $\left\{\operatorname{Form}^{\square}(R) \mid R \in \mathcal{R}\right\}$.

Proof. Let the normal description $S=\left\{\left(S_{R}^{i}, S_{=}^{i}\right)\right\}_{i \in I}$ be given.

In order to compute the disjunction form for the hypersequent rule induced by $S$, we first rewrite the rule as follows:

$$
\begin{gathered}
G \mid \cup_{j \in S_{R}^{i}} \otimes \Gamma_{j}^{\prime} \Rightarrow \quad\left(i \in I_{2}\right), \\
\frac{G \mid \cup_{j \in S_{R}^{i}} \otimes \Gamma_{j}^{\prime}, \cup_{j \in S_{=}^{i}} \Sigma_{j} \Rightarrow \cup_{j \in S_{=}^{i}} \Pi_{j} \quad\left(i \in I_{1}\right)}{G\left|\square \Gamma_{1}^{\prime}, \Sigma_{1} \Rightarrow \Pi_{1}\right| \cdots \mid \square \Gamma_{n}^{\prime}, \Sigma_{n} \Rightarrow \Pi_{n}} r(S) .
\end{gathered}
$$


In the above, we have partitioned $I=I_{1} \sqcup I_{2}$ such that $S_{=}^{i}=\emptyset$ iff $i \in I_{2}$. For the purpose of obtaining an association form as done in Section 5.2 we begin by reading $\square \Gamma_{j}^{\prime}$ and $\nabla \Gamma_{j}^{\prime}$ as the same multiset schematic-variable. Following that algorithm, the distinguished variable occurrences of each active component in the premises are: some $\Sigma_{j}\left(j \in S_{=}^{i}\right)$ for $i \in I_{1}$, and some $\nabla \Gamma_{j}^{\prime}\left(j \in S_{R}^{i}\right)$ for $i \in I_{2}$. Associating $\square \Gamma_{j}^{\prime}$ and $\otimes \Gamma_{j}^{\prime}$ to the same propositional variable, construct Form $(r(S))$ from the association form (Definition 19). The required formula $\operatorname{Form}^{\square}(r(S))$ is obtained by amending Form $(r(S))$ as follows:

(i) Replace every leading disjunct $B \wedge 1$ by $B$ (if $B$ is boxed) or $\square B$ (otherwise).

(ii) A $\square$ is placed in front of every propositional variable.

Now follow the proof for Form $(r(S))$ (Theorem 21) to prove that Form ${ }^{\square}(r(S))$ is a disjunction formula (provability, weakening, and splitting property). Regarding the amendments, observe that these can be emulated by applying the appropriate modal rules: for (i), use (4) to introduce a leading $\square$; for (ii), start with $\square p \Rightarrow \square p$ rather than $p \Rightarrow p$. We do have to account for our reading of $\square \Gamma_{j}^{\prime}$ and $\nabla \Gamma_{j}^{\prime}$ as the same variable despite these multiset schematic-variables having different instantiations (the former permits instantiation only by $\left\{\square A_{1}, \ldots, \square A_{k}\right\}$ and the latter only by $\left.\left\{A_{1}, \square A_{1}, \ldots, A_{k}, \square A_{k}\right\}\right)$. It turns out that this is not problematic because every instantiation of "strong box variable $\Rightarrow$ its box variable" and "box variable $\Rightarrow$ its strong box variable" is derivable in $\mathbf{S 4}$.

$$
\frac{\square(\wedge X) \Rightarrow \square(\wedge X)}{(\wedge X) \wedge \square(\wedge X) \Rightarrow \square(\wedge X)} \quad \frac{\frac{(\wedge X) \Rightarrow(\wedge X)}{\square(\wedge X) \Rightarrow(\wedge X)}(T) \quad \square(\wedge X) \Rightarrow \square(\wedge X)}{\square(\wedge X) \Rightarrow(\wedge X) \wedge \square(\wedge X)}
$$

To obtain a multiset-axiomatisation we follow the proof of Theorem 13. The only thing to check is that each disjunct in the disjunction form can be permuted downwards, i.e., adding this formula to the antecedents of the premise(s) and conclusion of a rule instance should not invalidate the rule instance. This holds for the instances of the (4) rule because this formula is boxed due to (i), and it is immediate for the other rules. Finally, a set-axiomatisation follows from the presence of contraction and weakening, refer to Lemma 24.

The above proof relies on the presence of the $(T)$ and (4) rules. In particular, if the (4) rule was replaced by the standard modal rule for $\mathbf{K}$, then the addition of a boxed formula to the premise and conclusion of the latter would invalidate the rule instance. This is why the theorem is framed with respect to $\mathbf{S 4}$.

Example 41. Consider $r\left(S_{\text {lin }}\right)$ in Example 39. Read $\square \Gamma_{1}^{\prime}$ and $\nabla \Gamma_{1}^{\prime}$ as the same variable, and read $\square \Gamma_{2}^{\prime}$ and $\nabla \Gamma_{2}^{\prime}$ as the same variable to obtain the association form. Associating $\square \Gamma_{1}^{\prime}$ and $\otimes \Gamma_{1}^{\prime}$ with the propositional variable $p$, and $\square \Gamma_{2}^{\prime}$ and $\nabla \Gamma_{2}^{\prime}$ with the propositional variable $q$, we obtain

$$
\operatorname{Form}\left(r\left(S_{\text {lin }}\right)\right):=((p \rightarrow q) \wedge 1) \vee((q \rightarrow p) \wedge 1) .
$$

Applying (i) we get $\square(p \rightarrow q) \vee \square(q \rightarrow p)$. After (ii) we obtain

$$
\operatorname{Form}^{\square}\left(r\left(S_{\text {lin }}\right)\right):=\square(\square p \rightarrow \square q) \vee \square(\square q \rightarrow \square p) \text {. }
$$


Let us prove that $\square(\square p \rightarrow \square q) \vee \square(\square q \rightarrow \square p)$ is a disjunction form of $r\left(S_{\text {lin }}\right)$. The following derivation in HS4 witnesses one half of the splitting property (the other derivation is analogous).

$$
\frac{\frac{\square \Gamma_{2}^{\prime} \Rightarrow \wedge\left(\square \Gamma_{2}^{\prime}\right)}{\square \Gamma_{2}^{\prime} \Rightarrow \square \wedge\left(\square \Gamma_{2}^{\prime}\right)}(4) \quad \frac{\nabla \Gamma_{1}^{\prime}, \Sigma_{2} \Rightarrow \Pi_{2}}{\square \wedge\left(\square \Gamma_{1}^{\prime}\right), \Sigma_{2} \Rightarrow \Pi_{2}}}{\frac{\square \wedge\left(\square \Gamma_{2}^{\prime}\right) \rightarrow \square \wedge\left(\square \Gamma_{1}^{\prime}\right), \square \Gamma_{2}^{\prime}, \Sigma_{2} \Rightarrow \Pi_{2}}{\square\left(\square \wedge\left(\square \Gamma_{2}^{\prime}\right) \rightarrow \square \wedge\left(\square \Gamma_{1}^{\prime}\right)\right), \square \Gamma_{2}^{\prime}, \Sigma_{2} \Rightarrow \Pi_{2}}}(T),(c),\left(\wedge_{L}\right)
$$

Observe that the instance of the right disjunct of the disjunction formula above is obtained by the substitution $q \mapsto \wedge \square \Gamma_{2}^{\prime}$ and $p \mapsto \wedge \square \Gamma_{1}^{\prime}$, analogous to the substructural case. The following establishes the provability property.

$$
\begin{gathered}
\frac{\triangle p \Rightarrow \square p \quad \nabla q \Rightarrow \square q}{\square p \Rightarrow \square q \mid \square q \Rightarrow \square p} r(S) \\
\frac{\Rightarrow \square p \rightarrow \square q \mid \Rightarrow \square q \rightarrow \square p}{\Rightarrow \square(\square p \rightarrow \square q) \mid \Rightarrow \square(\square q \rightarrow \square p)} \\
\frac{\Rightarrow \square(\square p \rightarrow \square q) \vee \square(\square q \rightarrow \square p) \mid \Rightarrow \square(\square p \rightarrow \square q) \vee \square(\square q \rightarrow \square p)}{\Rightarrow \square(\square p \rightarrow \square q) \vee \square(\square q \rightarrow \square p)}
\end{gathered}
$$

7.3. A new syntactic proof of analyticity for S5. A sequent calculus for S5 is obtained by the addition of the rules $(T)$ and (5) to the sequent calculus $\mathbf{L K}$ for classical propositional logic:

$$
\frac{\Gamma, A \Rightarrow \Delta}{\Gamma, \square A \Rightarrow \Delta}(T) \quad \frac{\square \Gamma \Rightarrow A, \square \Delta}{\square \Gamma \Rightarrow \square A, \square \Delta}(5) .
$$

In response to the failure of cut-elimination for this calculus, Takano [45] gave an intricate syntactic proof of analyticity, establishing that only cuts on subformulas are required. Prior to that, only a semantic argument had been shown, see [17].

A formula-axiomatisation for $\mathbf{S 5}$ can be established along the lines of Theorem 38 . Is it possible to tweak the methodology of this paper to obtain Takano's (stronger) analyticity result? The answer is affirmative, as shown below.

THEOREM 42. The sequent calculus for $\mathbf{S 5}$ is analytic.

Proof. Our starting point is a cut-free hypersequent calculus for $\mathbf{S 5}$ presented in [28]. It extends HS4 by the rule

$$
\frac{G \mid \square \Delta, \Gamma \Rightarrow \Pi}{G|\square \Delta \Rightarrow| \Gamma \Rightarrow \Pi}(M S) .
$$

This is a special case of Avron's rule $\left(M S_{A v}\right)$ (cf. Example 2). Cut-free HS4 $+(M S)$ derives exactly the same sequents as the sequent calculus for S5. Moreover, any instance of the rule $(M S)$ can be simulated using $(e c),\left(w_{l}\right)$, and using multiple instances of its single formula version $\left(M S_{1}\right)$ below: 


$$
\frac{G \mid \square A, \Gamma \Rightarrow \Pi}{G|\square A \Rightarrow| \Gamma \Rightarrow \Pi}\left(M S_{1}\right) .
$$

Consider a cut-free derivation $d$ in $\mathbf{H S 4}+\left(M S_{1}\right)$ of a sequent $S$. For simplicity, suppose that $d$ contains a single instance of $\left(M S_{1}\right)$ (in the general case, bottommost instances of $\left(M S_{1}\right)$ are eliminated at each step, refer to the proof of Theorem 13). From this instance, we can obtain the following derivations in HS4 $+\left(M S_{1}\right)$ :

$$
\frac{\square A \Rightarrow \square A}{G|\square A \Rightarrow \square A| \Gamma \Rightarrow \Pi, \square A} \text { (ew) } \quad \frac{G \mid \square A, \Gamma \Rightarrow \Pi}{G|\square A \Rightarrow| \square A, \Gamma \Rightarrow \Pi} \text { (ew). }
$$

Above left (right), the component $\square A \Rightarrow \square A$ (resp. $\square A, \Gamma \Rightarrow \Pi$ ) has a $\square A$ in the succedent (resp. antecedent) that was not present in the original derivation $d$. Proceed downward from each hypersequent mimicking the rules in $d$. Although these hypersequents each contain an additional occurrence of $\square A$ that needs to be propagated downwards, the same rule can be used for this purpose provided it is not (4). The issue with (4) is that it permits only a single formula in the succedent and the additional $\square A$ in the succedent would violate this. The solution is to use (5) instead. In this way we obtain $\left(M S_{1}\right)$-free hypersequent derivations (hence in HS5) of $\square A \# S$ and $S \# \square A$ (the latter denotes that $\square A$ is added to the succedent of $S$ ). Applying the cut-rule on $\square A$ on these sequents, we obtain a derivation of $S$ in HS5. Since $\square A$ occurred in the cut-free derivation $d$, it is a subformula of $S$. Since every rule in $\mathbf{H S 5}$ has one active component, we can extract an analytic derivation of $S$ in the sequent calculus for $\mathbf{S 5}$.

§8. Conclusions and open problems. We investigated generalisations of the subformula property that enabled us to retain the simplicity of the sequent calculus for various families of logics, and to establish meta-logical properties. This represents a departure from the typical approach in structural proof theory where the subformula property is treated as the primary goal, even if this means structural enrichment and new proof formalisms; think, e.g., the $\mid$ in the hypersequent calculus, the nesting of structural parentheses in the nested sequent calculus, or the display calculus [8] where each logical connective corresponds to a structural one. While the difficulties of dealing with an enriched structural language are well known, a formal assessment of this cost has never been investigated. Our transformations suggest a nuanced assessment method by pegging the strength of the subformula property in the hypersequent calculus to restrictions of the axiom instances. We aim to extend these transformations to formalisms beyond the hypersequent calculus such as the nested sequent (preliminary work along this line appears in [37]), display calculus, and bunched sequent calculus. This could pave the way for a new classification of logics and formalisms, according to the degree of boundedness of their boundedanalytic sequent calculi.

The starting point for the bounded-analytic sequent calculi presented here were the analytic hypersequent calculi obtained in [12, 28] from axiomatic extensions of Hilbert calculi, and those in [29] from adding frame conditions. This means that, implicitly, there is an algorithm transforming Hilbert calculi for these logics into bounded-analytic sequent calculi. How could we define a step-by-step 
transformation that takes a proof in the Hilbert calculus (or sequent calculus proofs with arbitrary cuts) and yields a bounded-analytic sequent calculus without taking a detour through another formalism? That is, from arbitrary cuts to cuts on specific axiom instances. Cut-elimination is the bedrock of structural proof theory and what we are proposing here is a new paradigm: cut-restriction, with cut-elimination as a special case.

The perspective using logical embeddings allowed us to abstract from the minute details of the proof theory. This was exploited to obtain new complexity results for contractive-mingle substructural logics, and to situate earlier results within our theory. Investigating the correspondence between embedding functions and their amenability to meta-theoretic argument (think decidability, complexity, interpolation, and so on) could lead to boundedness-specific methods that are applicable over a range of different logics.

Acknowledgments. Work supported by the FWF projects I 2982, P32684-N, and P33548. Ramanayake would like to acknowledge the financial support of the CogniGron research center and the Ubbo Emmius Funds (University of Groningen).

\section{REFERENCES}

[1] A. Avellone, U. Moscato, P. Miglioli, and M. Ornaghi, Generalized tableau systems for intermediate propositional logics, Automated Reasoning with Analytic Tableaux and Related MethodsTABLEAUX 1997 (D. Galmiche, editor), Springer, New York, 1997, pp. 43-61.

[2] A. Avron, A constructive analysis of RM, this Journal, vol. 52 (1987), no. 4, pp. 939-951.

[3] - Hypersequents, logical consequence and intermediate logics for concurrency. Annals of Mathematics and Artificial Intelligence, vol. 4 (1991), no. 3-4, pp. 225-248.

[4] - The method of hypersequents in the proof theory of propositional non-classical logics, Logic: From Foundations to Applications (Staffordshire, 1993) (W. Hodges, M. Hyland, C. Steinhorn, and J. Truss, editors), Oxford Science Publications, Oxford University Press, New York, 1996, pp. 1-32.

[5] - Non-deterministic semantics for families of paraconsistent logics, Handbook of Paraconsistency (J.-Y. Beziau and W. Carnielli, editors), College Publications, London, 2007, pp. 285-320.

[6] A. Avron and O. LAHAV, A unified semantic framework for fully structural propositional sequent systems. ACM Transactions on Computational Logic, vol. 14 (2013), no. 4, pp. 1-33.

[7] A. R. Balasubramanian, T. Lang, and R. Ramanayake, Decidability and complexity in weakening and contraction hypersequent substructural logics, LICS 2021, accepted for publication.

[8] N. D. Belnap, JR., Display logic. Journal of Philosophical Logic, vol. 11 (1982), no. 4, pp. 375-417.

[9] C. Benzmüller, Cut-elimination for quantified conditional logic. Journal of Philosophical Logic, vol. 46 (2017), no. 3, pp. 333-353.

[10] N. BEZHANISHVILI and S. GHILARDI, The bounded proof property via step algebras and step frames. Annals of Pure and Applied Logic, vol. 165 (2014), no. 12, pp. 1832-1863.

[11] N. Bezhanishvili, S. Ghilardi, and F.M. Lauridsen, One-step Heyting algebras and hypersequent calculi with the bounded proof property. Journal of Logic and Computation, vol. 27 (2016), no. 7, pp. 2135-2169.

[12] A. Ciabattoni, N. Galatos, and K. Terui, From axioms to analytic rules in nonclassical logics, LICS 2008: Proceedings of the 2008 23rd Annual Symposium on Logic in Computer Science, IEEE, New York, 2008, pp. 229-240.

[13] - Algebraic proof theory: Hypersequents and hypercompletions. Annals of Pure and Applied Logic, vol. 168 (2017), no. 3, pp. 693-737.

[14] A. Ciabattoni, T. LANG, and R. Ramanayake, Bounded sequent calculi for non-classical logics via hypersequents, Automated Reasoning with Analytic Tableaux and Related Methods-TABLEAUX 2019 (S. Cerrito and A. Popescu, editors), Springer, New York, 2019, pp. 94-110. 
[15] M. D'Agostino and M. Mondadori, The taming of the cut. Classical refutations with analytic cut. Journal of Logic and Computation, vol. 4 (1994), no. 3, pp. 285-319.

[16] H. A. Feitosa and I. M. L. D’Ottaviano, Conservative translations. Annals of Pure and Applied Logic, vol. 108 (2001), no. 1-3, pp. 205-227.

[17] M. FitTing, Subformula results in some propositional modal logics. Studia Logica, vol. 37 (1978), no. 4, pp. 387-391.

[18] N. Galatos, P. Jipsen, T. Kowalski, and H. Ono, Residuated Lattices: An Algebraic Glimpse at Substructural Logics, Studies in Logic and the Foundations of Mathematics, vol. 151, Elsevier, Amsterdam, 2007.

[19] N. Galatos and H. Ono, Algebraization, parametrized local deduction theorem and interpolation for substructural logics over FL. Studia Logica, vol. 83 (2006), no. 1-3, pp. 279-308.

[20] G. Gentzen, Untersuchungen über das logische Schließen. 39:176-210, 405-431, 1934/35, English translation in The Collected Papers of Gerhard Gentzen (M. E. Szabo, editor), North-Holland, Amsterdam, 1969, pp. 68-131.

[21] R. Horcík and K. Terui, Disjunction property and complexity of substructural logics. Theoretical Computer Science, vol. 412 (2011), no. 31, pp. 3992-4006.

[22] T. Hosor, On intermediate logics, this Journal, vol. 36 (1971), pp. 329-330.

[23] — Pseudo two-valued evaluation method for intermediate logics. Studia Logica: An International Journal for Symbolic Logic, vol. 45 (1986), no. 1, pp. 3-8.

[24] E. JERÁBEK, The ubiquity of conservative translations. The Review of Symbolic Logic, vol. 5 (2012), no. 4, pp. 666-678.

[25] - A note on the substructural hierarchy. Mathematical Logic Quarterly, vol. 62 (2016), no. $1-2$, pp. $102-110$.

[26] N. KAMIDE, Substructural logics with mingle. Journal of Logic, Language and Information, vol. 11 (2002), no. 2, pp. 227-249.

[27] T. KowAlski and H. Ono, Analytic cut and interpolation for bi-intuitionistic logic. The Review of Symbolic Logic, vol. 10 (2017), no. 2, pp. 259-283.

[28] H. Kurokawa, Hypersequent calculi for modal logics extending S4, New Frontiers in Artificial Intelligence (Y. Nakano, K. Satoh, and D. Bekki, editors), Springer, New York, 2014, pp. 51-68.

[29] O. LAHAV, From frame properties to hypersequent rules in modal logics, LICS 2013: Proceedings of the 2013 28th Annual ACM/IEEE Symposium on Logic in Computer Science, IEEE, New York, 2013, pp. $408-417$.

[30] O. LahaV and Y. Zohar, On the construction of analytic sequent calculi for sub-classical logics, WoLLIC 2014 (U. Kohlenbach, P. Barceló, and R. de Queiroz, editors), Springer, Berlin, 2014, pp. 206-220.

[31] B. Lellmann, Hypersequent rules with restricted contexts for propositional modal logics. Theoretical Computer Science, vol. 656 (2016), pp. 76-105.

[32] B. Lellmann and D. Pattinson, Cut elimination for shallow modal logics, Automated Reasoning with Analytic Tableaux and Related Methods-TABLEAUX 2011 (K. Brünnler and G. Metcalfe, editors), Springer, New York, 2011, pp. 211-225.

[33] E. Marchioni and F. Montagna, On triangular norms and uninorms definable in $Ł \Pi \frac{1}{2}$. International Journal of Approximate Reasoning, vol. 47 (2008), no. 2, pp. 179-201.

[34] G. Metcalfe and F. Montagna, Substructural fuzzy logics, this Journal, vol. 72 (2007), no. 3 , pp. 834-864.

[35] G. Metcalfe, N. Olivetti, and D. Gabbay, Proof Theory for Fuzzy Logics, Springer Series in Applied Logic, vol. 39, Springer, New York, 2009.

[36] G. E. MinTs, Some calculi of modal logic. Trudy Matematicheskogo Instituta imeni VA Steklova, vol. 98 (1968), pp. 88-111.

[37] E. Pimentel, R. Ramanayake, and B. Lellmann, Sequentialising nested systems, Proceedings of the Automated Reasoning with Analytic Tableaux and Related Methods-TABLEAUX 2019 (S. Cerrito and A. Popescu, editors), Springer, Cham, 2019, pp. 147-165.

[38] G. PotTinger, Uniform, cut-free formulations of T, S4 and S5 (abstract), this Journal, vol. 48 (1983), no. 3, p. 900.

[39] R. Ramanayake, Extended Kripke lemma and decidability for hypersequent substructural logics, LICS 2020: Proceedings of the 35th Annual ACM/IEEE Symposium on Logic in Computer Science (H. Hermanns, L. Zhang, N. Kobayashi, and D. Miller, editors), IEEE, New York, 2020, pp. 795-806. 
[40] K. SASAKI, The simple substitution property of the intermediate propositional logics. Bulletin of the Section of Logic, vol. 18 (1989), no. 3, pp. 94-99.

[41] - The simple substitution property of Gödel's intermediate propositional logics $S_{n}$ 's. Studia Logica, vol. 49 (1990), no. 4, pp. 471-481.

[42] K. SASAKI, The simple substitution property of the intermediate propositional logics on finite slices. Studia Logica, vol. 52 (1993), no. 1, pp. 41-62.

[43] R. M. Smullyan, Analytic cut, this Journal, vol. 33 (1968), pp. 560-564.

[44] G. Sт John, Decidability for residuated lattices and substructural logics, Electronic theses and dissertations, 2019.

[45] M. TAKANO, Subformula property as a substitute for cut-elimination in modal propositional logics. Mathematica Japonica, vol. 37 (1992), pp. 1129-1145.

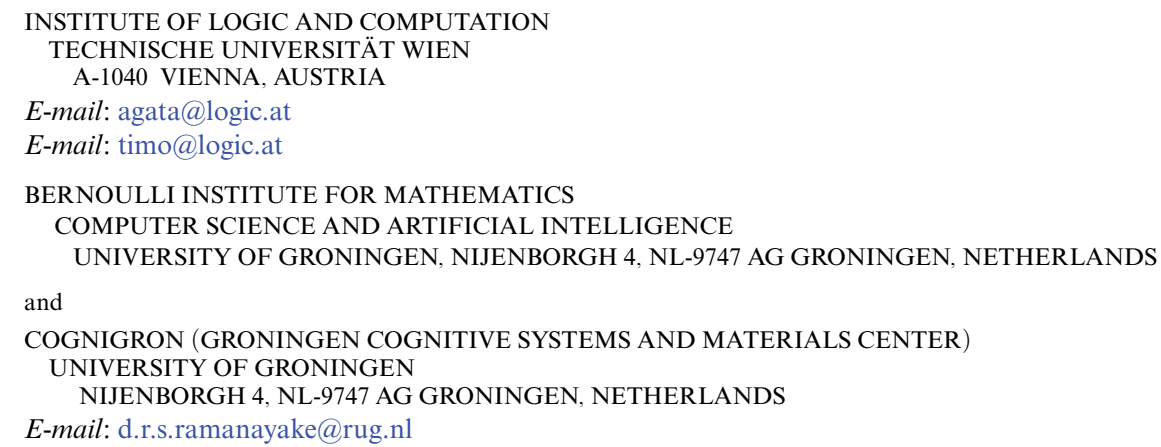

